\title{
The Effect of Plasma Shape and Neutral Beam Mix on the Rotation Threshold for RMP-ELM Suppression
}

\author{
C. Paz-Soldan, ${ }^{1}$ R. Nazikian, ${ }^{2}$ L. Cui, ${ }^{2}$ B. C. Lyons,${ }^{1}$ D. M. \\ Orlov $^{3}$, A. Kirk, ${ }^{4}$ N. C. Logan, ${ }^{2}$ T. Osborne, ${ }^{1}$ W. Suttrop, ${ }^{5}$ D. \\ Weisberg, ${ }^{1}$ \\ ${ }^{1}$ General Atomics, P.O. Box 85608 San Diego, California 92186-5608, USA \\ 2 Princeton Plasma Physics Laboratory, Princeton, New Jersey 08543, USA \\ ${ }^{3}$ University of California San Diego, La Jolla, California 92093-0417, USA \\ ${ }^{4}$ Culham Center for Fusion Energy, Culham, United Kingdom \\ ${ }^{5}$ Max Planck Institute for Plasma Physics, Garching, Germany
}

\begin{abstract}
Varying the neutral beam injection (NBI) mix reveals a clear pedestal-top rotation threshold for edge localized mode (ELM) suppression by resonant magnetic perturbations. Guided by expectations for the RMP penetration mechanism, the rotation threshold is found to correspond to a critical radius for the ExB rotation zero-crossing. No such critical radius is observed for the electron perpendicular rotation zero-crossing. Varying the amount and ratio of power in different NBI source geometries (termed the NBI mix) also reveals that the rotation threshold can be crossed at widely varying total injected NBI torques. Computing the local torque density at the edge, the rotation threshold is found to be crossed when the local edge NBI torque is negative in nearly all discharges. Reducing the upper triangularity from the ITERsimilar value of 0.3 to 0.1 significantly reduces the pedestal height and width. This in turn: 1) Increases the rotation threshold and yields a more outward critical ExB rotation zero-crossing location. 2) Decreases the density threshold, consistent with a comparable collisionality range at lower pedestal temperatures. 3) Increases the input torque requirement, due to observed lower confinement and smaller intrinsic torque. These findings represent an important step along the road to predicting ELM suppression access conditions in future tokamaks such as ITER, where the toroidal rotation is expected to be low and consequently the rotation zero-crossing far from the pedestal-top.
\end{abstract}




\section{Introduction and Motivation}

Effective control of the edge-localized mode (ELM) is an essential aspect of operating fusion-grade tokamaks such as ITER [1, 2]. One approach for ELM control is the use of resonant magnetic perturbations (RMPs), a technique which has found wide application in the tokamak research program worldwide [3, 4, 5, 6, 7, 8, 9]. While mitigation of the ELM by RMPs is ubiquitous, a more effective yet more challenging operating mode is that of full ELM suppression, characterized by low pedestal collisionality and narrow resonant windows in the safety factor. Recent work has increased confidence that this regime is achievable on ITER and beyond: DIII-D results have demonstrated compatibility of ELM suppression with many of the ITER 15 MA Q $=10$ scenario conditions, namely the normalized current, pressure, and shape - albeit with some degradation of confinement [8]. Further, alongside DIII-D [3] full ELM suppression is now routinely achieved in KSTAR [10], EAST [9], and recently ASDEX-Upgrade (AUG) [11, 12]. Interestingly, ELM suppression on AUG was only possible by raising the upper triangularity $\left(\Delta_{\text {up }}\right)$ to above normal values for that device [12, 11, 13], partially motivating the studies described herein.

Worldwide exploration of RMP-ELM suppression has yielded significant insights into the underlying physical mechanisms and resultant optimization strategies. Penetration of the applied RMPs [14] at edge rational surfaces, while not unanimously agreed upon, is a leading contender to explain the pedestal bifurcation into the ELM suppressed state. This is most readily determined by considering the fast-time scale variations at the threshold of ELM suppression. Bifurcations of the plasma flow together with changes of the non-axisymmetric magnetic field structure are observed on ms-time scales and are consistent with expectations of the field penetration effect [15, 16, 17]. Comparing conditions in and out of ELM suppression with longer analysis intervals reveals the same effect, in particular when considering changes in the toroidal and poloidal phase of the magnetic field structure [9, 18]. Fluctuations are also robustly found to increase (on both the fast and slow time scale) likely due to changes in the ExB shearing rate induced by the penetration effect [15, 16, 19, 20]. These fluctuations may ultimately play an important role in enhancing the pedestal-top transport.

In parallel, dedicated RMP spectral optimization studies have converged on the crucial role of maximizing resonant coupling to the edge rational surfaces [21, 7, 22], and if conditions require it, maximizing edge resonant coupling while holding core resonant coupling to a sufficiently low value to avoid penetration of the core resonant surfaces [10, 23, 24]. Though the reason $\Delta_{\text {up }}$ was so effective in AUG is not yet resolved, an increase in edge resonant coupling may partially explain this result. This is because at high $\Delta_{\text {up }}$ in AUG the pedestal pressure and bootstrap current both significantly increased - both of which have been identified as a significant drive to resonant coupling and edge deformations [16, 25].

Amidst this generally positive picture, recent DIII-D experiments have highlighted a potentially serious compatibility issue for ITER: if the input torque (and rotation) is 
reduced through counter- $I_{P}$ directed neutral beam injection (NBI), ELM suppression is robustly lost (though ELM mitigation remains) [26]. By comparing flow profiles before and after the loss of ELM suppression, a radially inward shift of the low-flow region of both the ExB rotation $\left(E_{r} / R B_{p} \equiv \omega_{E}\right)$ and the perpendicular electron flow $\left(\omega_{\perp, e}\right)$ profiles was found. Note that these two flows are separated by the electron diamagnetic flow $\left(\omega_{*, e}\right)$, according to: $\omega_{\perp, e}=\omega_{E}+\omega_{*, e}$. Linear single-fluid MHD (considering $\omega_{E}$ ) and two-fluid MHD (considering $\omega_{\perp, e}$ ) modeling with the M3D-C1 code [27] found a radially inward shift of the field penetration region, as would be expected based on the basic paradigm that low flow begets field penetration. While this paradigm is not in doubt, open questions remain concerning which flow $\left(\omega_{E}\right.$ or $\left.\omega_{\perp, e}\right)$ must be low to allow field penetration. Two-fluid MHD theory prescribes $\omega_{\perp, e}$ as the relevant flow [28, 27], possibly with a further correction for the electron thermal force [29]. Single-fluid MHD theory prescribes $\omega_{E}$ as the relevant flow, though there is no doubt that the two-fluid model is more accurate than its single-fluid predecessor. Gyrokinetic treatments however have also found that low $\omega_{E}$ flow can enable field penetration due to particle resonance effects 30].

Experimentally, detailed information is lacking on which flow profile is best correlated with ELM suppression, how low the flow must be, and how close to the pedestal-top the low-flow condition must be met. These questions are of great importance since the expected locations of the low-flow regions for these profiles can vary significantly. This is especially true in large tokamaks such as ITER, where low normalized momentum injection should yield a low $\omega_{E}$, yet high electron pressure gradients should yield a large $\omega_{*, e}$. Thus, the $\omega_{E}$ and $\omega_{\perp, e}$ profiles would be expected to be very different from each-other and scale pessimistically from their values in present devices. As the plasma shape (ie, $\Delta_{\text {up }}$ ) affects the pedestal pressure and thus the diamagnetic flows separating $\omega_{E}$ and $\omega_{\perp, e}$, the shaping effect is also a useful tool to understand the extrapolation of ELM suppression to ITER and beyond.

In this paper, variation of the amount and ratio of power in different NBI source geometries (termed the NBI mix) reveals a pedestal-top rotation threshold for RMPELM suppression that is found to correspond to a critical radius for the $\omega_{E}$ zero-crossing. In contrast, no critical zero-crossing location is observed for the $\omega_{\perp, e}$ zero-crossing. This threshold is examined as a function of pedestal structure actuated by the plasma shape. Decreasing $\Delta_{\text {up }}$ from 0.3 to 0.1 is found to narrow and lower the pedestal, causing an increase of the rotation threshold together with a more outward critical $\omega_{E}$ zerocrossing location. Lowering $\Delta_{\text {up }}$ also decreases the density threshold, consistent with a comparable collisionality range at lower pedestal temperatures. Varying the NBI mix also also reveals that the rotation threshold can be crossed at widely varying total injected NBI torques. Computing the local torque density at the edge, the rotation threshold is found to be crossed when the local edge NBI torque is negative in all but one discharge. Notwithstanding the NBI torque profile effect, reducing $\Delta_{\text {up }}$ generally increases the input torque requirement, due to observed lower confinement and expected smaller intrinsic torque in the weaker shape. 


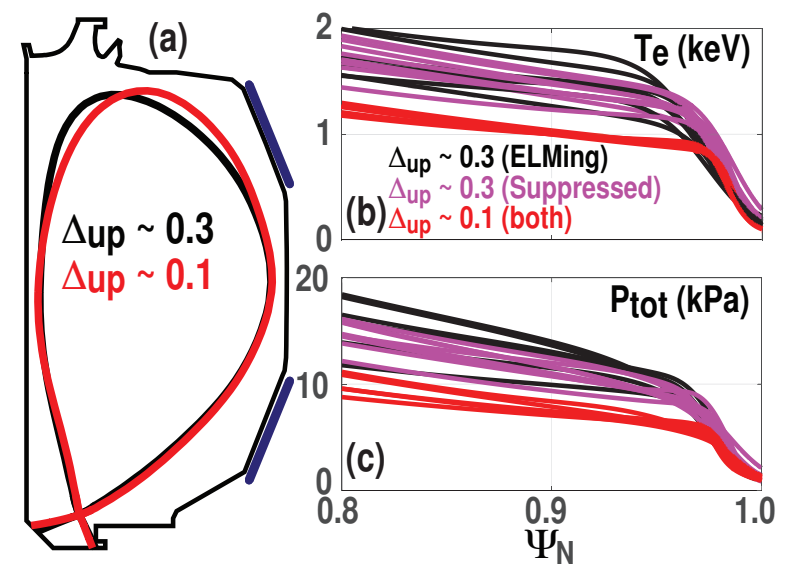

Figure 1. (color online) (a) Cross-section of the plasma shapes used in this study. Typical pedestal (b) electron temperature profiles and (c) total pressure profiles for both $\Delta_{\text {up }}$. At $\Delta_{\text {up }} \sim 0.1$ the profiles are largely unchanged when entering ELM suppression, unlike at $\Delta_{\text {up }} \sim 0.3$.
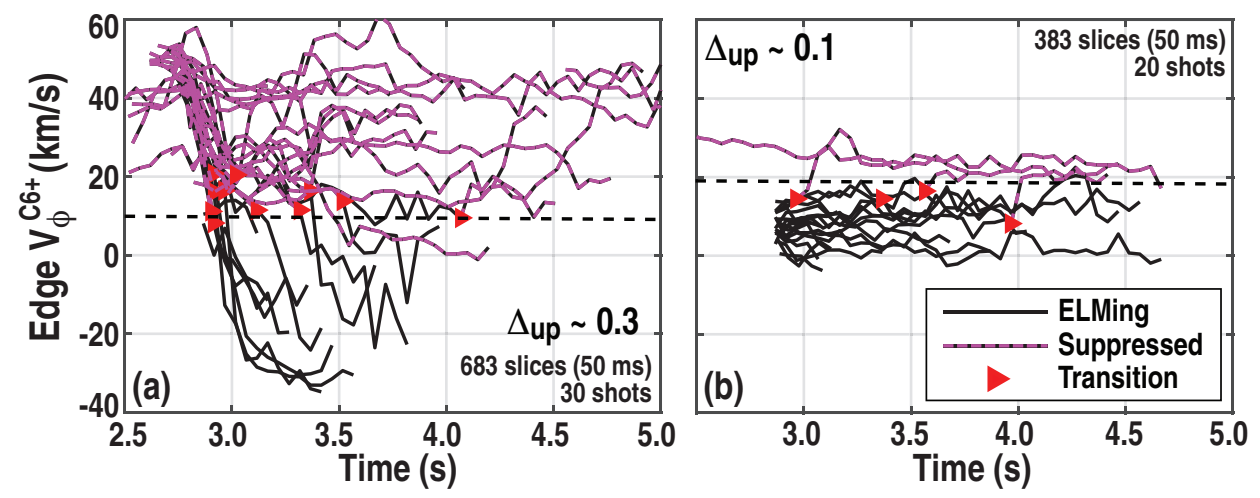

Figure 2. (color online) Observation of an edge impurity toroidal rotation $\left(V_{\phi}^{C 6+}\right)$ threshold for RMP-ELM suppression in (a) the $\Delta_{\text {up }} \sim 0.3$ shape, and (b) the $\Delta_{\text {up }} \sim 0.1$ shape. The threshold is higher in the $\Delta_{\text {up }} \sim 0.1$ shape. RMP current is constant during this interval.

This paper is structured as follows. First, in Section 2, discharge conditions and experimental execution are described alongside a first presentation of the rotation threshold. Section 3 discusses the rotation threshold for the ITER-similar shape $\left(\Delta_{\text {up }} \sim 0.3\right)$ and isolates the role of the $\omega_{E}$ and $\omega_{\perp, e}$ zero-crossing. Section 4 discusses how varying $\Delta_{\text {up }}$ can dramatically change the operating space for ELM suppression, both in density and rotation. Section 5 relates the rotation threshold to the NBI torque, both globally and locally, and discusses the role of plasma confinement. A summary of results and discussion are given in Section 6, and conclusion in Sec. 7. Appendix A discusses the impact of rotation on the pedestal shape and thus the pedestal stability, leading to a potential alternate path to the ELM instability. 


\section{Experimental Setup and Basic Observations}

These experiments were designed to isolate the rotation threshold and its dependence on input NBI geometry and plasma shaping.

Discharges were conducted in two plasma shapes with varying upper triangularity $\left(\Delta_{\text {up }}\right)$, shown in Fig. 1. The stronger shape, with $\Delta_{\text {up }} \sim 0.3$ is the canonical 'ITERsimilar shape' used in numerous RMP-ELM suppression studies on DIII-D [8, 15, 31, 32]. This discharge well-matches the ITER cross-sectional shape and $\Delta_{\text {up }}$, but has a lower aspect ratio than ITER plasmas (2.8 vs. 3.1) to better match the DIII-D vacuum vessel geometry. To vary the pedestal structure and explore this effect on the observed thresholds, a lower $\Delta_{\text {up }}$ discharge was developed with $\Delta_{\text {up }} \sim 0.1$. A cross-section comparison of the two explored plasma shapes is shown in Fig. 1. The lower half and divertor geometry were intentionally held constant, with $\Delta_{\text {down }} \approx 0.6$ for both.

Both shapes had the same plasma current $\left(I_{P}\right)$ of $1.6 \mathrm{MA}$, and toroidal field $\left(B_{T}\right)$ of $1.96 \mathrm{~T}$. As such, the safety factor $\left(q_{95}\right)$ of the two shapes varied, with the $\Delta_{\text {up }} \sim 0.3$ shape yielding $q_{95}=3.5$ while in the $\Delta_{\text {up }} \sim 0.1$ shape $q_{95}=3.2$. Conveniently, these two $q_{95}$ values were $\approx 1 / 3$ integer apart and as such both existed within a sufficiently robust resonant window in $q_{95}$ for $n=3$ RMPs. A detailed study of the $q_{95}$ window dependence with plasma shape was not attempted. No effort was made to match the global $\beta_{N}$, which varied from 1.2-2.3 at $\Delta_{\text {up }} \sim 0.3$ and from 1.2-1.6 at $\Delta_{\text {up }} \sim 0.1$. As subsequent analysis did not reveal any dependence on core parameters, this work only discusses the pedestal.

As can be seen in Fig. 1 $(\mathrm{b}, \mathrm{c})$, variations of $\Delta_{\text {up }}$ successfully yielded very different pedestal shapes. The pedestal-top electron temperature $\left(T_{\mathrm{e} \text {,ped }}\right)$ was particularly sensitive, dropping by $\approx 40 \%$ as $\Delta_{\text {up }}$ was reduced. Near the threshold for ELM suppression in each shape, the total pressure was reduced by a comparable amount.

The RMP spectrum used throughout these experiments was an in-vessel $n=3$ 'even parity' configuration, delivering strong resonant coupling to the edge rational surfaces. The RMP coil current amplitude was held constant within each shape, at $3.0 \mathrm{kA}$ for the $\Delta_{\text {up }} \sim 0.3$ (vacuum $B_{\text {res }} \approx 5 \mathrm{G}$ ) shape and $3.5 \mathrm{kA}$ (vacuum $B_{\text {res }} \approx 6 \mathrm{G}$ ) for the $\Delta_{\text {up }} \sim 0.1$ shape. The slight amplitude increase at $\Delta_{\text {up }} \sim 0.1$ was found to be helpful to access robust ELM suppression for the wall conditions of the $\Delta_{\text {up }} \sim 0.1$ experimental session.

Variations of the NBI mix were performed to identify and isolate the rotation threshold. This entailed changing the ratio of tangentially directed to radially directed NBI sources, the ratio of of co- $I_{P}$ directed and counter- $I_{P}$ directed NBI sources, and the injected voltage of the NBI sources independently. Various combinations were tried to modify the rotation profile in different ways based on the expected differences in the torque profile for these different combinations. Furthermore, a powerful new technique to dynamically vary the NBI voltage within the discharge [33, 34] was utilized to more smoothly cross the observed rotation threshold.

Varying the NBI mix was found to be a very effective way to cross the rotation 
threshold in both shapes. The basic observation explored in this work is shown in Fig. 2 . With few exceptions, when the edge carbon impurity toroidal rotation $\left(V_{\phi}^{C 6+}\right)$ decreases below $\approx 10 \mathrm{~km} / \mathrm{s}$ in the $\Delta_{\text {up }} \sim 0.3$ shape, ELM suppression is lost. This measurement is from a single location in space. Since the plasma shape is held constant, the fixed location maps to a fixed radius near the pedestal-top. In the $\Delta_{\text {up }} \sim 0.1$ shape, when edge $V_{\phi}^{C 6+}$ increases above $\approx 20 \mathrm{~km} / \mathrm{s}$ ELM suppression is found. Note rotation data in Fig. 2 is averaged over $50 \mathrm{~ms}$, giving rise to some scatter in the transition threshold which will be better resolved with analysis presented later in this paper. As can be seen, during the analysis period the $\Delta_{\text {up }} \sim 0.3$ discharges go from high to low rotation and exit ELM suppression, whereas the $\Delta_{\text {up }} \sim 0.1$ discharges go from low to high rotation and enter ELM suppression. This different intial condition is due to an errant 30 -ms duration NBI off-period at $2.8 \mathrm{~s}$ in the $\Delta_{\text {up }} \sim 0.1$ discharges, which very rapidly reduced the rotation and transitioned these discharges to the low rotation ELMing state prior to the main analysis period. This in principle opens the door to hysteresis effects varying the observed rotation threshold. However, taking that the rotation is the directly controlling parameter for field penetration, hysteresis would only be expected in the actuators yielding a given rotation profile, as opposed to the rotation iself.

\section{Resolving the Rotation Threshold in the ITER-similar $\left(\Delta_{\text {up }} \sim 0.3\right)$ shape}

This section will focus on resolving the dynamics and origin of the rotation threshold in the $\Delta_{\text {up }} \sim 0.3$ shape. Differences with $\Delta_{\text {up }}$ are left to Sec. 4 while the role of the NBI mix is left to Sec. 5. First, time-dependent analysis of the $\omega_{E}$ and $\omega_{\perp, e}$ profiles will be presented, identifying several phases in the rotation evolution prior to the loss of ELM suppression. A criticality condition in the $\omega_{E}$ flow will be presented, while no correlation with the $\omega_{\perp, e}$ flow will be found. These results will be interpreted in terms of linear MHD modeling.

\subsection{Time-dependent Evolution of Zero-Crossings}

A new time-dependent profile fitting tool [35] is used to evaluate the $\omega_{E}$ and $\omega_{\perp, e}$ profile over the duration of the discharge as the plasmas of Fig. 2(a) evolve through the rotation threshold. The electron profiles are measured through Thomson Scattering (TS) and are ELM phase averaged during ELMing periods, taking intervals of $50-90 \%$ of the inter-ELM cycle. Electron profiles are aligned to the separatrix by enforcing $T_{\mathrm{e} \text {,sep }}$ $=80 \mathrm{eV}$. This value originates from parallel transport arguments to the divertor for the typical connection lengths of DIII-D [36]. Ion profiles are measured via charge exchange recombination (CER) spectroscopy, which is capable of measuring both the toroidal and poloidal flows necessary to calculate $\omega_{E}$. Due to ambiguities over how to shift the ion profiles to the separatrix in the presence of RMP fields, no shifting is applied to the ion profiles shown in this section. Thus, the $\Psi_{N}$ ordinate can equivalently be considered a real-space ordinate, since the plasma shape was held constant as the 

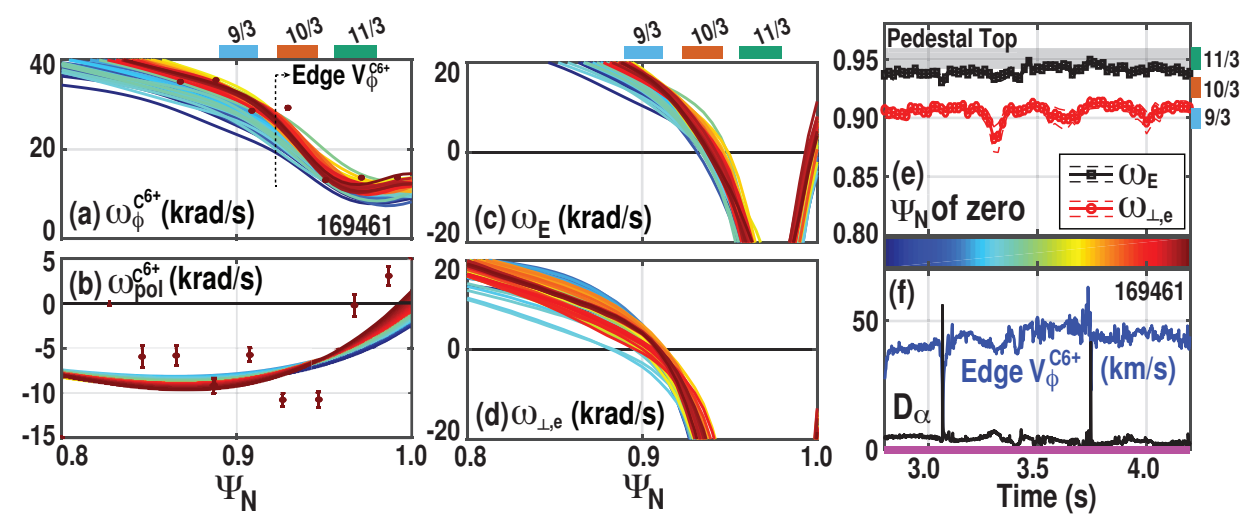

Figure 3. (color online) Profile evolution for high-torque ELM-suppressed discharge 169461. Profiles of (a) toroidal flow $\left(\omega_{\phi}^{C 6+}\right.$, with example CER data), (b) poloidal flow ( $\omega_{\text {pol }}^{C 6+}$, with example CER data), (c) $\omega_{E}$, (d) $\omega_{\perp, e}$ are summarized in terms of (e) the $\Psi_{N}$ location of the zero-crossing. For reference, (f) shows the time-evolution of edge $V_{\phi}^{C 6+}$ and $D_{\alpha}$. Location of 'Edge $V_{\phi}^{C 6+}$ ' as used throughout this paper is indicated in (a), where $V_{\phi}^{C 6+}=R \omega_{\phi}^{C 6+}$ and $R \approx 2.2 \mathrm{~m}$. Estimates of the rational surface locations from kinetic equilibrium reconstructions are given by colored boxes in this and subsequent figures.

rotation evolved. Profiles are made every $20 \mathrm{~ms}$, aggregating raw data points within each averaging interval (TS every $2.5 \mathrm{~ms}$, and CER every 2-5 ms).

Before exploring dynamics as rotation is reduced, it is instructive to consider a discharge for which the input torque and thus the rotation remains elevated. The profile evolution for this sort of discharge is shown in Fig. 3(a-d) for toroidal flow $\left(\omega_{\phi}^{C 6+}\right.$ $\left.=V_{\phi}^{C 6+} / \mathrm{R}\right)$, poloidal flow $\left(\omega_{\text {pol }}^{C 6+}=-V_{\text {pol }}^{C 6+} B_{T} /\left|R B_{p}\right|\right), \omega_{E}$, and $\omega_{\perp, e}$. Representative $\omega_{\phi}^{C 6+}$ and $\omega_{\text {pol }}^{C 6+}$ CER data are included for the final time slice (note the profile fit is also constrained by data outside the plotted $\Psi_{N}$ interval). This information is more simply presented in Fig. 3(e), where only the $\Psi_{N}$ location of the $\omega_{E}$ and $\omega_{\perp, e}$ zero-crossing is tracked in time. This ELM-suppressed discharge maintains high rotation and an outward zero-crossing location for both the $\omega_{E}$ and $\omega_{\perp, e}$ profile. Uncertainty in the zero-crossing is hereafter obtained by mapping a $3 \mathrm{krad} / \mathrm{s}$ error bound in $\omega_{E}$ and $\omega_{\perp, e}$ to the corresponding change in the zero-crossing, as indicated by a dashed line (barely visible in Fig. 3[e] but larger in subsequent discharges). Three $\mathrm{krad} / \mathrm{s}$ is used as it is thought to be a reasonable estimate of systematic uncertainties due to unaccounted for atomic physics corrections in the analysis of CER spectra. Equilibrium reconstructions show that the $\omega_{E}$ zero-crossing is roughly aligned with the $m / n=11 / 3$ rational surface while the $\omega_{\perp, e}$ zero-crossing is roughly aligned with the $9 / 3$ surface, and both are 'near' the pedestal top. As this is the condition used for nearly all RMP-ELM suppression experiments on DIII-D, it is easy to see why ambiguity exists as to whether the $\omega_{E}$ or $\omega_{\perp, e}$ zero-crossing best correlates with ELM suppression.

Slowly increasing the voltage (and power) of a counter- $I_{P}$ directed NBI source using the technique described in Sec. 2 enables a gradual transition through the rotation threshold as summarized in Fig. 4. Three distinct phases within the ELM suppressed 


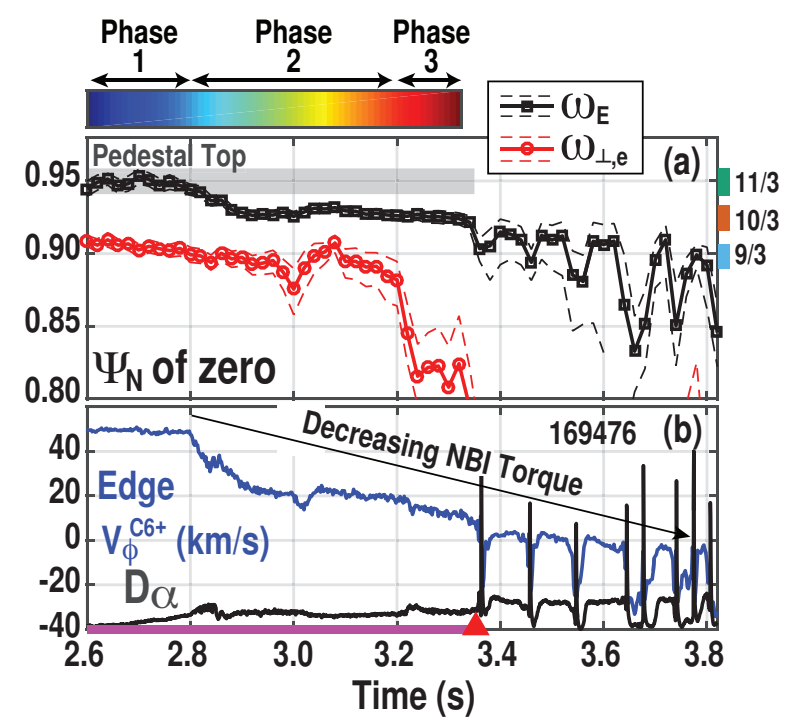

Figure 4. (color online) $\Psi_{N}$ location of the zero-crossings as input torque is gradually reduced and the rotation threshold is crossed. After the first ELM, a dithering phase from 3.4 to $3.6 \mathrm{~s}$ is observed where the plasma repeatedly regains ELM suppression, until later more regular ELMing observed.

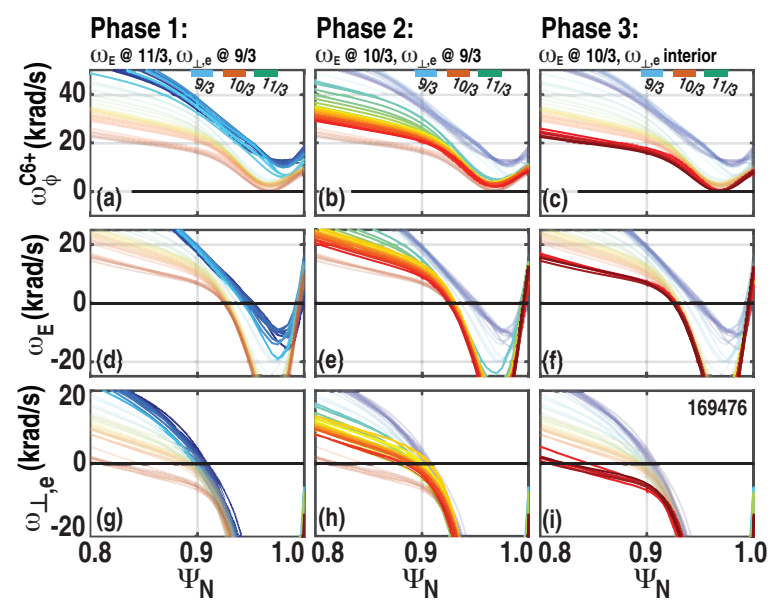

Figure 5. (color online) Rotation profile comparison for the three phases identified as input torque is gradually reduced and the rotation threshold is crossed. Colors indicate the time in the discharge as given by the color bar in Fig. 4 Data from all phases is included in each plot, with only the active phase indicated by thicker opaque lines.

period are identified, and are highlighted in Fig. 5 and above the colorbar of Fig. 4. Phase 1 is equivalent to the conditions of the discharge of Fig. 3 and corresponds to fairly high momentum input and rotation. As torque is reduced the rotation falls and the $\omega_{E}$ zero-crossing shifts inwards, roughly from the location of the $11 / 3$ to the $10 / 3$ surface. This discrete shift of the $\omega_{E}$ profile is commonly observed in these discharges, despite the gradual reduction of the input torque. Figure 5 shows that $\omega_{\phi}^{C 6+}$ interior of the pedestal also falls gradually. This suggests that if the field penetration is tracking $\omega_{E}$, the field penetration region shifts from the $11 / 3$ surface to the $10 / 3$ surface. As 

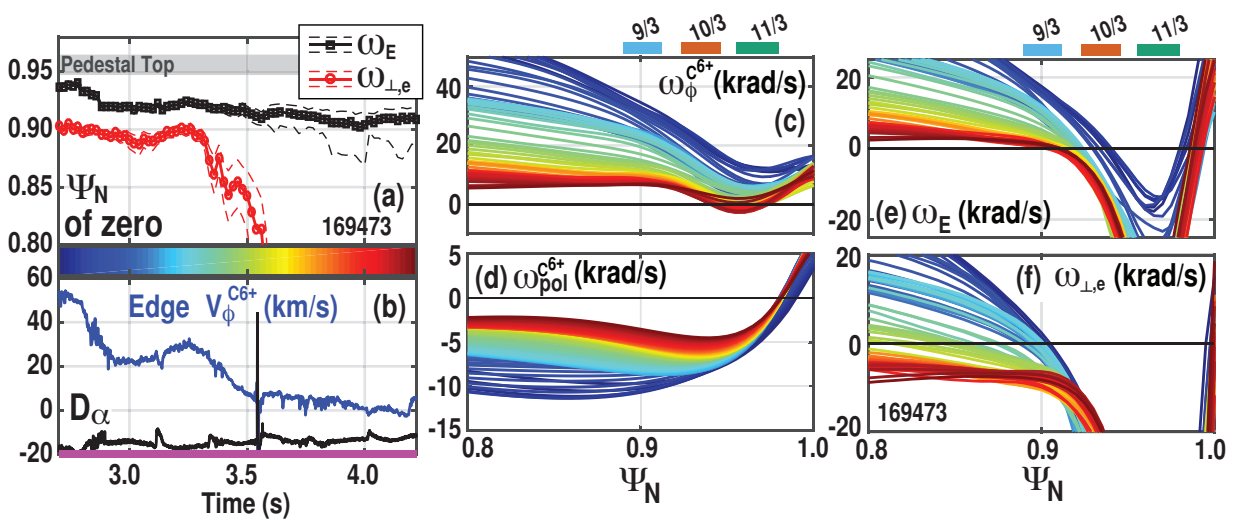

Figure 6. (color online) Profile evolution of anomalously low $V_{\phi}^{C 6+}$ discharge 169473. (a) An exterior $\omega_{E}$ zero-crossing is maintained despite the absence of a zero-crossing in the $\omega_{\perp, e}$ profile. (b) Long-duration ELM suppression is maintained during this interval (until a locked-mode forms). Profiles of (c) $\omega_{\phi}^{C 6+}$, (d) $\omega_{\text {pol }}^{C 6+}$, (e) $\omega_{E}$, (f) $\omega_{\perp, e}$ used to obtain the zero-crossing positions.

seen in both Figs. 4 and 5, the flow changes between Phase 1 and Phase 2 do not shift the location of the $\omega_{\perp, e}$. As the torque and rotation fall further, a Phase 3 is identified during which the $\omega_{E}$ zero-crossing is unchanged but the $\omega_{\perp, e}$ zero-crossing abruptly moves far interior from the pedestal. Despite this large motion of the $\omega_{\perp, e}$ zero, ELMs do not promptly return. No indications from the $D_{\alpha}$ recycling light or the behavior of the pedestal parameters are found that indicate ELM suppression has been lost during this Phase. Specifically, $n_{\mathrm{e}, \text { ped }}$ and $T_{\mathrm{e} \text {,ped }}$ remain stationary despite the change in the $\omega_{\perp, e}$ zero-crossing location. Finally, at the end of Phase 3, ELMs return, preceded by a rapid reduction of the rotation not easily captured by the $20 \mathrm{~ms}$ averaging utilized for this analysis. For brevity $\omega_{\text {pol }}^{C 6+}$ is not included in Fig. 5 as only a gradual monotonic decrease is found. These dynamics are consistent with the observations of Ref. [26] except that in these discharges (at $q_{95}=3.5$ instead of 3.2), two rational surfaces are compatible with ELM suppression, as opposed to only one in Ref. [26]. As such, in these discharges the bistable nature of the zero-crossing can be observed without exiting ELM suppression.

The ELMing period after $3.4 \mathrm{~s}$ is unusual in that dithering-type behavior is seen, with the pedestal evidently regaining brief periods of ELM suppression before losing it again in a cyclic manner. This behavior is very reminiscent of DIII-D experience with $n=2$ RMPs as in Ref. [15], but is very unusual for $n=3$ RMPs on DIIID. During the periods of enhanced recycling and apparent ELM suppression, the $\omega_{E}$ zero-crossing returns to its original outward position (within the time-resolution of this analysis technique).

The previous discharge was highlighted in great detail because it happened to spend roughly equal times in each Phase. Considering the full dataset, discharges can spend an arbitrary length of time in each phase depending on how the input torque and rotation evolve. The discharge of Fig. 3, for example, exists entirely in Phase 

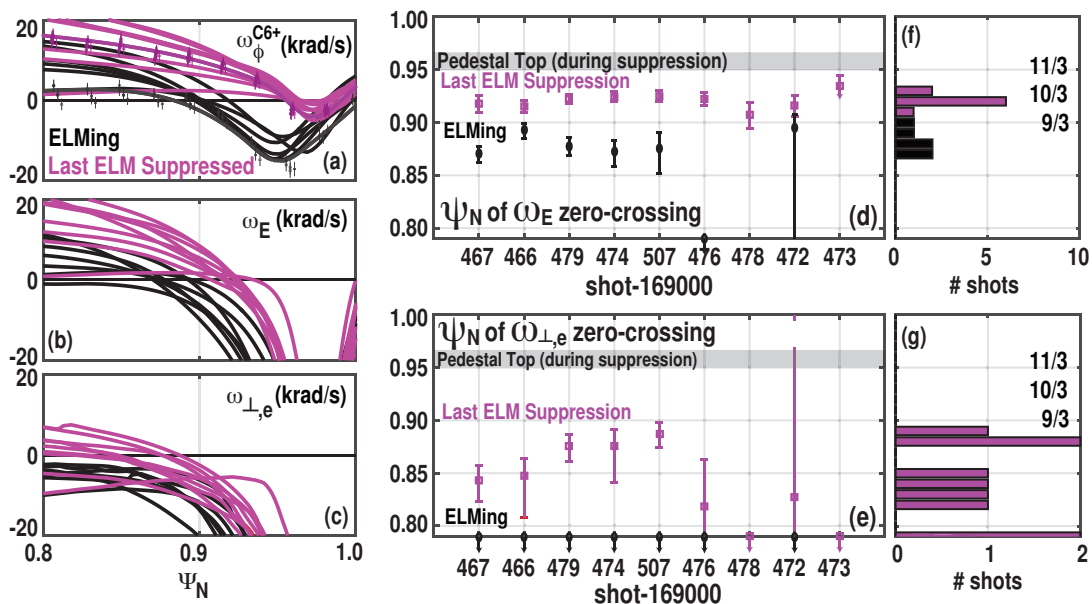

Figure 7. (color online) Profile analysis as the rotation threshold is crossed demonstrating a critical $\omega_{E}$ radius. Profiles of (a) $\omega_{\phi}^{C 6+}$ (with example CER data), (b) $\omega_{E}$, (c) $\omega_{\perp, e}$ before and after ELM suppression is lost. The $\Psi_{N}$ position of the (d) $\omega_{E}$ and (e) $\omega_{\perp, e}$ zero-crossing for each discharge that crosses the threshold, along with $(\mathrm{f}, \mathrm{g})$ histograms of the zero-crossing location.

1. Interestingly, discharges which persist in Phase 3 can also be identified. One such discharge is presented in Fig. 6, where nearly one second elapses in Phase 3, with the zero-crossing of $\omega_{\perp, e}$ very far from the pedestal top. This discharge is also of interest because it is the single magenta time trace below the raw $V_{\phi}^{C 6+}$ threshold shown in Fig. 2(a). As can be seen in Fig. 6, while this discharge has anomalously low $V_{\phi}^{C 6+}$, its $\omega_{E}$ zero-crossing is still firmly outwards, due to a significantly weaker $\omega_{*, C}$.

\subsection{Observation of Critical ExB Zero-Crossing Location}

The discharges highlighted in Section 3.1 support the interpretation that the $\omega_{\perp, e}$ zerocrossing is not the controlling parameter for the loss of ELM suppression as the rotation is reduced, since long-duration ELM suppressed periods are obtained with very interior $\omega_{\perp, e}$ zero-crossings. Figure 7 presents profile reconstruction using a dedicated pedestal fitting tool [37] for all discharges in Fig. 2(a) that cross the rotation threshold. For extra precision, this tool allows slight radial shifting of the CER data (of $1 \%$ or less in $\Psi_{N}$ ) which is employed to keep the $T_{\text {i }}$ profile minimum at the location of the separatrix. Unfortunately, this tool does not directly propagate uncertainties into the computed $\omega_{E}$ or $\omega_{\perp, e}$ profile. As such zero-crossing error bars in Fig. 7 are estimated by mapping a $3 \mathrm{krad} / \mathrm{s}$ uncertainty in the underlying CER data to a corresponding uncertainty in that profile's zero-crossing. Consideration of multiple discharges also serves to inform the variability in the underlying data and fitting. Instead of continuously constructing profiles every $20 \mathrm{~ms}$, the last available analysis period before ELM suppression is lost is contrasted to the first available analysis period in the ELMing phase. Immediately after the last available ELM suppressed profile reconstruction, the rotation falls further and the anomalous pedestal transport is lost. This is evidenced by a fall in the $D_{\alpha}$ 


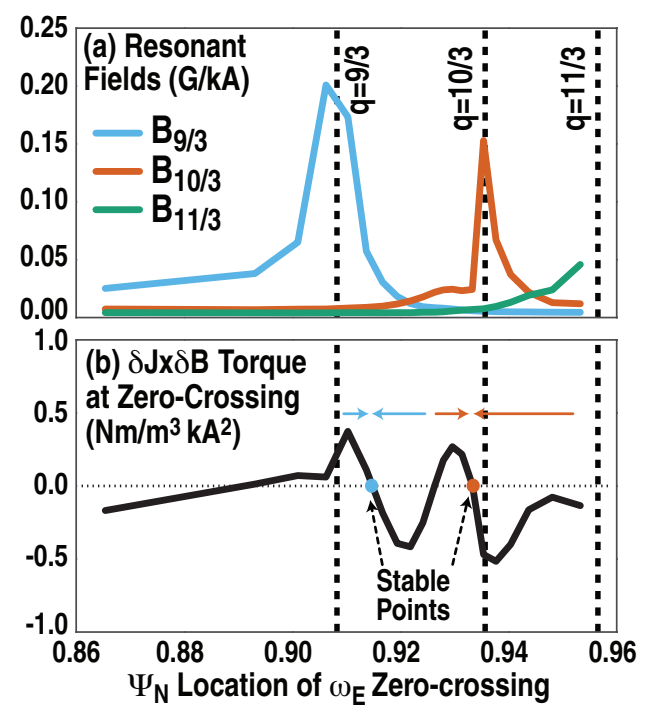

Figure 8. (color online) Dependence of (a) resonant fields at their rational surface (indicating penetration) and (b) quasi-linear local $\delta J \times \delta B$ torque on $\omega_{E}$ zero-crossing location for an interpolated set of $\omega_{E}$ profiles from Fig. 5 . Colored arrows in (b) indicate the direction the torque would shift the $\omega_{E}$ zero-crossing location, and demonstrate the existence of stable points near the rational surfaces.

baseline and increase in $n_{\text {e,ped }}$ and $T_{\text {e,ped }}$ on short time scales, with the ELM coming shortly thereafter. As such, these profiles would be expected to identify any criticality condition in the pedestal profiles.

As shown in Fig. 7(a), the $\omega_{\phi}^{C 6+}$ profile is significantly different in ELMing and ELM suppression, as expected from Fig. 2(a). Note that no systematic difference is observed in the measured $\omega_{\text {pol }}^{C 6+}$ profiles, and as such they are not included in Fig. 7 for brevity. There is a remarkable consistency in the $\omega_{E}$ zero-crossing location for the critical final profile before ELM suppression is lost [Fig. 7(b)]. This is presented systematically in Fig. $7(\mathrm{~d})$, where the $\omega_{E}$ zero-crossing location is plotted as a function of discharge number, and as a histogram in Fig. 7(f). The histogram reveals the final $\omega_{E}$ zero-crossing locations at the exit from ELM suppression are narrowly clustered at $\Psi_{N}$ of 0.92 , roughly the location of the $10 / 3$ resonant surface.

Considering $\omega_{\perp, e}$ in the same manner, no such similarity is found across discharges. Discharges lose ELM suppression with a very wide range of $\omega_{\perp, e}$ zero-crossing locations, and ELM suppression can be maintained with the $\omega_{\perp, e}$ zero-crossing inside of $\Psi_{N}=0.8$. Interestingly, all ELMing cases have an $\omega_{\perp, e}$ zero-crossing inside of $\Psi_{N}=0.8$. This analysis thus serves to demonstrate the importance of the $\omega_{E}$ profile and in particular the location of the $\omega_{E}$ zero-crossing location over the $\omega_{\perp, e}$ profile. The implications of this will be described in Sec. 6, and the the relationship of the critical $\omega_{E}$ zero-crossing to the input NBI torque will be discussed in Section 5 . 


\subsection{Interpretation from Linear MHD Modeling}

An interpretation for the above observations is provided by single-fluid linear MHD modeling using the M3D-C1 code [27]. Single-fluid MHD modeling is used to match the observed importance of the $\omega_{E}$ flow, though a complete treatment will likely require both two-fluid and kinetic resonance effects as will be discussed in Sec. 6. The amount of field penetration and associated quasi-linear $\delta J \times \delta B$ torque is computed for an interpolated set of $21 \omega_{E}$ profiles spanning the time interval shown in Fig. 5. Interpolation is done to assure a fairly uniform spacing of the $\omega_{E}$ zero-crossing location in $\Psi_{N}$. Furthermore, to isolate the rotation effect, the background equilibrium is held constant and corresponds to ELM suppressed conditions for these experiments. Note the absence of experimental profiles with zero-crossings exterior to the $11 / 3$ surface prevents the extension of the computation to this region. The most outward zero-crossing is within experimental uncertainty of the $11 / 3$ surface.

The amount of field penetration and associated local torque is plotted for each of the $21 \omega_{E}$ profiles is shown in Fig. 8, indexed by the $\omega_{E}$ zero-crossing location for each profile. As can be seen, the maximum field penetration at a given rational surface occurs when the $\omega_{E}$ zero-crossing is aligned with the rational surface location. This is consistent with the Ohm's law in single-fluid M3D-C1 modeling. If the $\omega_{E}$ zero-crossing is interior (exterior) of a nearby rational surface, the $\delta J \times \delta B$ torque is positive (negative) and this would tend to force the $\omega_{E}$ zero-crossing outwards (inwards). In this way, despite the input torque being reduced, it would be expected for the $\omega_{E}$ zero-crossings to linger at the stable points near rational surface locations, and then more discretely transition to an interior surface when local torque balance is lost. Interestingly, as will be shown in Sec. 5, the computed local $\delta J \times \delta B$ torque about an order of magnitude larger than the NBI torque density at the edge. Also note the stable points are slightly offset from the rational location due to non-ideal effects included in M3D-C1 [38].

These results are fully consistent with previously published results in Ref. [38] using $n=2$ fields, but here are extended to $n=3$ to match the conditions of these experiments. What is novel in these cases is that at penetration at both the $11 / 3$ and 10/3 surface appears to be compatible with ELM suppression, while the $9 / 3$ surface is

not. This is in contrast to recent results with $n=2$ fields [15] where penetration at the $8 / 2$ surface was compatible yet penetration at the $7 / 2$ surface was not. What sets the critical distance between the pedestal-top and the penetrated rational surface is not known.

\section{Modifying the ELM Suppression Operating Space via the Pedestal Shape}

The previous section resolved the rotation threshold in terms of the $\omega_{E}$ zero-crossing location focusing on the $\Delta_{\text {up }} \sim 0.3$ discharges. This section will consider how varying $\Delta_{\text {up }}$ changes the rotation threshold and the critical $\omega_{E}$ zero-crossing location. Additionally, other observed variations of the ELM suppression operating space with 

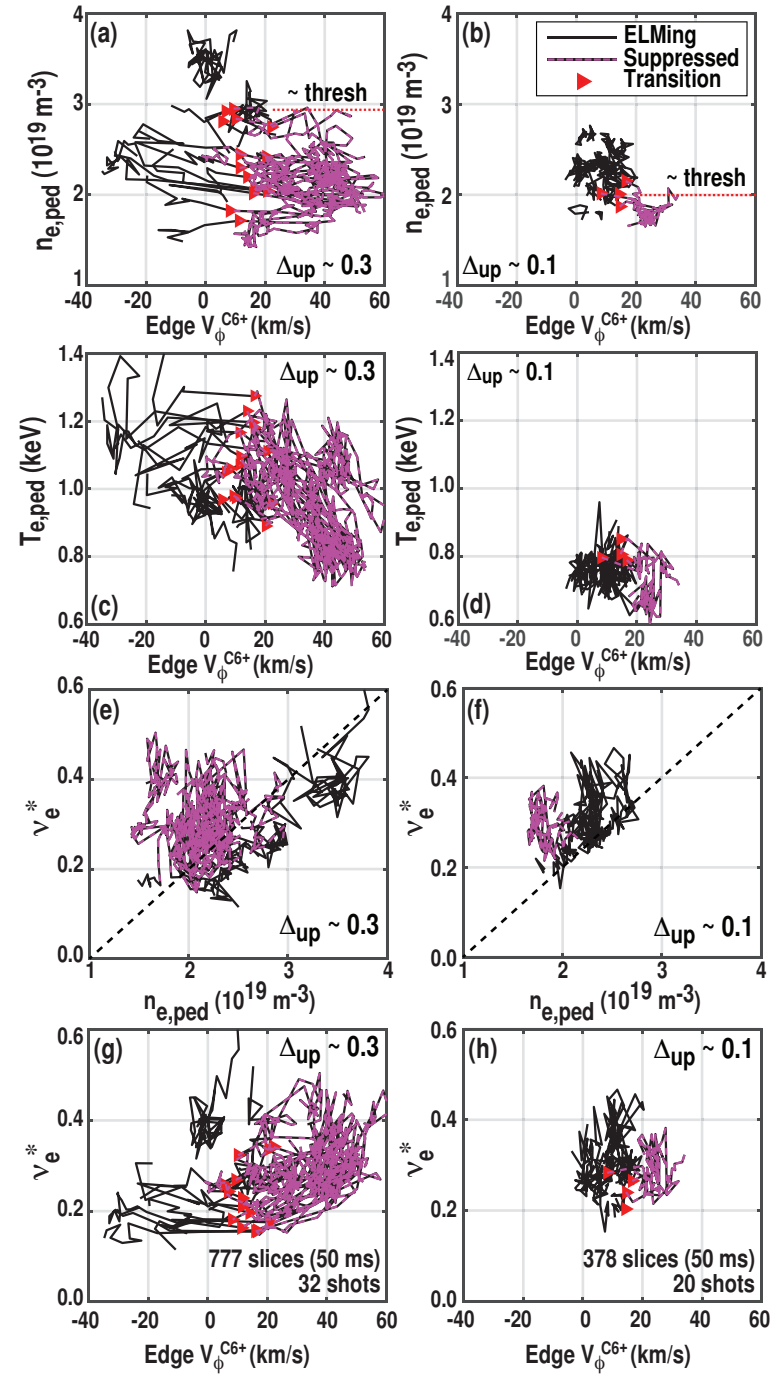

Figure 9. (color online) Variation of ELM suppression operating space with upper triangularity $\left(\Delta_{\text {up }}\right)$, considering the controlling variables of $n_{\text {e,ped }}, T_{\text {e,ped }}$, edge $V_{\phi}^{C 6+}$, and $\nu_{e}^{*}$. The operating space is significantly reduced for $\Delta_{\text {up }} \sim 0.1$ as compared to $\Delta_{\text {up }} \sim 0.3$. Data is averaged over $50 \mathrm{~ms}$.

$\Delta_{\text {up }}$ will be described, namely the pedestal-top density $\left(n_{\mathrm{e}, \text { ped }}\right)$, electron temperature $\left(T_{\text {e,ped }}\right)$ and collisionality $\left(\nu_{e}^{*}\right)$ calculated using the formula in Ref. [39].

\subsection{Variation of ELM Suppression Operating Space with $\Delta_{u p}$}

Figure 9] demonstrates the operational space for ELM suppression in a variety of parameters, contrasting findings in each $\Delta_{\text {up }}$. For this full-dataset analysis, $n_{\mathrm{e}, \mathrm{ped}}$ and $T_{\text {e,ped }}$ are calculated using automated tanh fits and edge $V_{\phi}^{C 6+}$ is from a single pedestaltop CER chord. As can be seen in Figs. $9(\mathrm{a}, \mathrm{b})$, the $n_{\mathrm{e}, \mathrm{ped}}$ operating space is very different. As such, a separate $\Delta_{\text {up }} \sim 0.3$ high $n_{\text {e,ped }}$ dataset was added to Fig. 9 (not considered in Sec. 3) to better compare the $n_{\mathrm{e}, \mathrm{ped}}$ limit in both shapes. This was necessary because all 
the discharges of Sec. 3 operated well below the empirical $n_{\mathrm{e} \text {,ped }}$ limit, owing to the lowrecycling wall conditions present during that session. Taking these additional discharges into account, the $n_{\text {e,ped }}$ threshold is shown to be reduced from just below $3 \times 10^{19} \mathrm{~m}^{-3}$ to $\approx 2 \times 10^{19} \mathrm{~m}^{-3}$ when $\Delta_{\text {up }}$ is reduced from 0.3 to 0.1 . Below these $n_{\text {e,ped values, ELM }}$ suppression is still precluded if the edge $V_{\phi}^{C 6+}$ is too low. Interestingly, no data points were found with $n_{\text {e,ped }}$ higher than its threshold and $V_{\phi}^{C 6+}$ rotating significantly faster than its threshold. To operate in this region of parameter space would require levels of input torque not accessible in these experiments, as will be discussed in Sec. 5 .

Figures $9(\mathrm{c}, \mathrm{d})$ demonstrate that the significant differences in the operating space are observed alongside a significantly lower $T_{\text {e,ped }}$. The reduction of $T_{\text {e,ped }}$ is expected from the variation of the kink-peeling limit with $\Delta_{\text {up }}$ [38]. Beyond the large $T_{\text {e,ped }}$ differences between plasma shapes, the $\Delta_{\text {up }} \sim 0.3$ dataset [Fig. [9(c)] reveals an interesting correlation of $T_{\text {e,ped }}$ with edge $V_{\phi}^{C 6+}$. Higher $T_{\text {e,ped }}$ is correlated with reduced $V_{\phi}^{C 6+}$. The origin of this correlation is unknown but will be given special attention in Appendix A.

The reduction of the $n_{\text {e,ped }}$ threshold observed alongside a decreased $T_{\text {e,ped }}$ operating point suggests that both discharges operate in a similar collisionality range, as is confirmed in Figs. 9(e,f). ELM suppression exists generally with $\nu_{e}^{*}$ below 0.4 , though no clear threshold is found. Interestingly, a lower boundary in $\nu_{e}^{*}$ seemingly appears. These datapoints however are the low $V_{\phi}^{C 6+}$ datapoints of Figs. 9(a,b), which due to the increased $T_{\mathrm{e} \text {,ped }}$ at low $V_{\phi}^{C 6+}$ operate at comparatively lower $\nu_{e}^{*}$. For completeness, the datasets are represented in terms of $\nu_{e}^{*}$ and edge $V_{\phi}^{C 6+}[\mathrm{Figs} 9(\mathrm{~g}, \mathrm{~h})]$, indicating the high-level observations: 1) The very different $n_{\mathrm{e}, \text { ped }}$ threshold maps onto comparable $\nu_{e}^{*}$ values, and 2) the edge $V_{\phi}^{C 6+}$ threshold is dominant and neatly separates the datasets into ELMing and ELM suppressed.

\subsection{Operating Space of $n_{e, p e d}$ and $\omega_{E}$ Zero-Crossing Location}

The basic operating space shown in Fig. 9 is that of $n_{\text {e,ped }}$ and edge $V_{\phi}^{C 6+}$, where $n_{\text {e,ped }}$ is calculated using automated tanh fits made at each TS laser pulse and edge $V_{\phi}^{C 6+}$ is from a single pedestal-top chord. Dedicated pedestal profile analysis again using the tool described in Ref. [37] is now undertaken of discharge time-slices found in various areas of this operating space, as shown in Fig. 10(a,b), and separated by whether the profiles were ELM suppressed or not. Uncertainties in $n_{\text {e,ped }}$ and edge $V_{\phi}^{C 6+}$ are taken as the standard deviation over the profile analysis interval. ELMing profiles are separated into two types: cases where $V_{\phi}^{C 6+}$ or $n_{\text {e,ped }}$ was firmly away from the threshold (black squares), and 'marginal' cases where either $n_{\text {e,ped }}$ or edge $V_{\phi}^{C 6+}$ was near-threshold (cyan diamonds). The marginality of these conditions is confirmed by noting that in every occasion the plasma entered ELM suppression less than one energy confinement time after the profile reconstruction was made. Again, the discharges of Sec. 3 are included in Fig. $10(\mathrm{a}, \mathrm{c})$ and are found well below the empirical $n_{\mathrm{e}, \text { ped }}$ limit.

A critical location of the $\omega_{E}$ zero-crossing is found for both $\Delta_{\text {up }}$ values, shown in Fig. 10(c,d), although it is shifted significantly outwards in the $\Delta_{\text {up }} \sim 0.1$ shape. This 

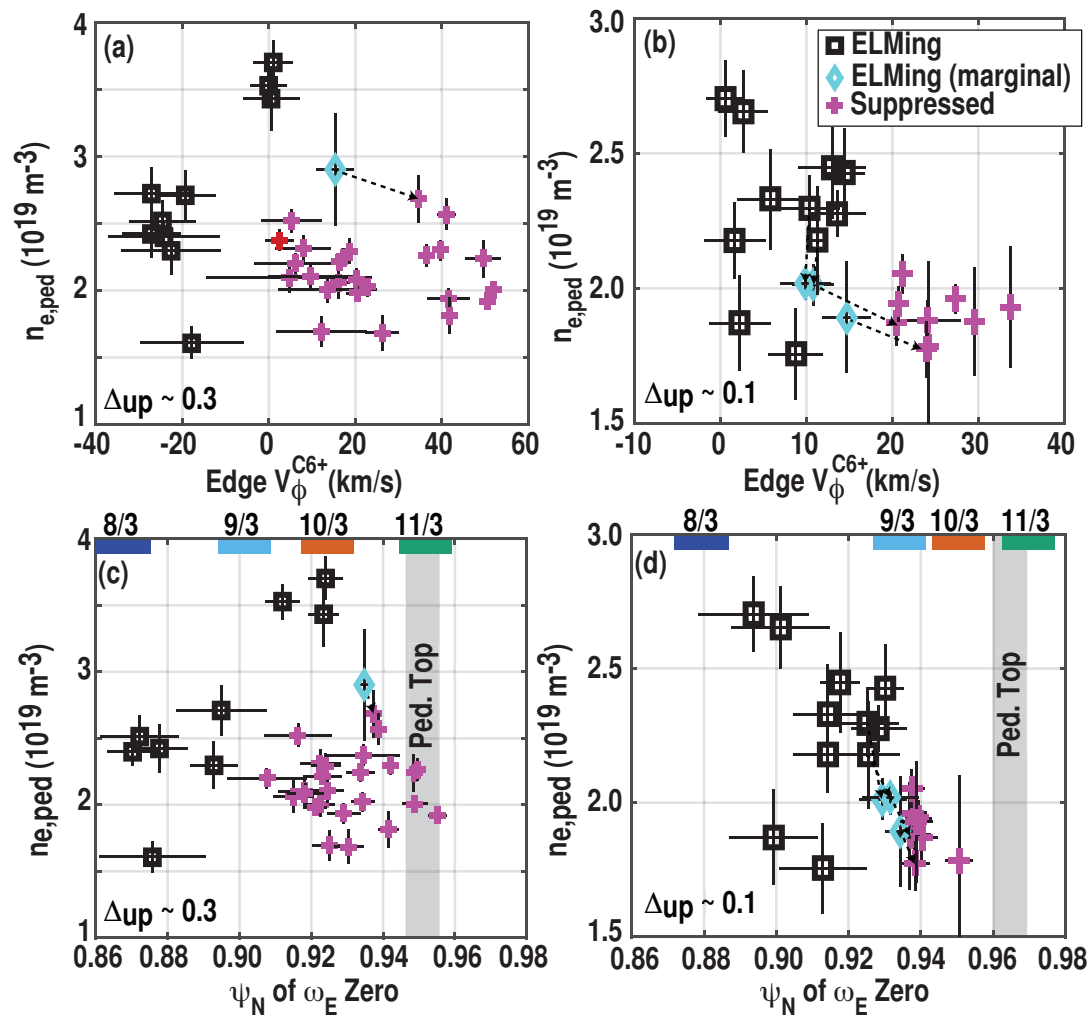

Figure 10. (color online) Comparison of operating space plot for both $\Delta_{\text {up }}$ in terms of $(\mathrm{a}, \mathrm{b})$ basic observables and $(\mathrm{c}, \mathrm{d})$ profile-derived $\omega_{E}$ zero-crossing location. ELMing and suppressed profiles are differentiated, with marginal ELMing indicating a bifurcation into ELM suppression immediately after the analysis period. The critical $\omega_{E}$ zerocrossing location shifts outward at low $\Delta_{\text {up }}$.

finding relates the higher rotation threshold in the $\Delta_{\text {up }} \sim 0.1$ shape to a different $\Psi_{N}$ location of the $\omega_{E}$ zero-crossing, roughly consistent with the location of the $9 / 3$ rational surface. The reason for this more outward zero-crossing location is not known, but a hypothesis is provided in Sec. 6.1. As before, zero-crossing error bars are estimated by mapping a $3 \mathrm{krad} / \mathrm{s}$ uncertainty in the $\omega_{E}$ profile to a corresponding uncertainty in the zero-crossing.

Comparing this analysis to that of Sec. 2 and 3 two distinctions are noted. First, a few $\Delta_{\text {up }} \sim 0.3$ cases are found with edge $V_{\phi}^{C 6+}$ slightly below the threshold shown in Sec. $2(\approx 5 \mathrm{~km} / \mathrm{s}$ in Fig. 10 a] vs. $\approx 10 \mathrm{~km} / \mathrm{s}$ in Fig. $2[\mathrm{a}])$. This small difference arises from a shorter averaging interval used in this analysis $(20 \mathrm{~ms})$ vs. that of Fig. 2 (50 ms), with Fig. 10 thus being more accurate. Second, the discrete nature of the $\omega_{E}$ zero-crossing found in Sec. 3.1 and interpreted in Sec. 3.3 is not as clear in Fig. 10.(c). This is thought to be due to small variations in the rational surface location between the dozen discharges contained in Fig. 10(c) that act to smear out this effect. 


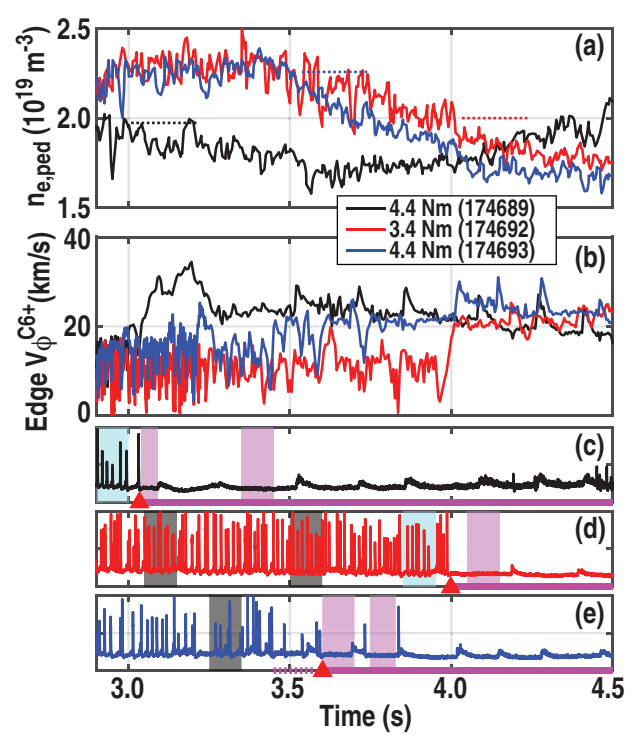

Figure 11. (color online) Uncontrolled (fixed RMP current) evolution of $\Delta_{\text {up }} \sim 0.1$ discharges towards ELM suppression, in terms of (a) $n_{\mathrm{e}, \mathrm{ped}}$, (b) edge $V_{\phi}^{C 6+}$, and (c,d,e) $D_{\alpha}$. Colored bands on $(\mathrm{c}, \mathrm{d}, \mathrm{e})$ correspond to datapoints with dashed lines in Fig. 10. b).

\subsection{Closer examination of Density-Threshold Dynamics at Low $\Delta_{u p}$}

Also of interest when considering the low $\Delta_{\text {up }}$ cases of Fig. 10(d) is the location of the 'marginal' profiles (those that are about to enter ELM suppression, yet are still ELMing). At low $\Delta_{\text {up }}$, these marginal points have very similar $\omega_{E}$ zero-crossing location as the ELM suppressed discharges. It stands to reason then that these profiles should have already been ELM-suppressed based solely on operating space considerations.

The evolution of these discharges as they evolve towards ELM suppression is shown in Fig. 11, corresponding to the arrows in Figs. 10(b,d). The key evolving parameter in the low $\Delta_{\text {up }}$ discharges is $n_{\text {e,ped }}$, which slowly decays after stopping a gas bleed at $3.5 \mathrm{~s}$ in the two higher $n_{\mathrm{e}, \mathrm{ped}}$ discharges of Fig. 11. During the $n_{\mathrm{e}, \mathrm{ped}}$ decay, pedestal temperatures are unchanged as expected for operation on the kink-peeling pedestal stability boundary. As $n_{\mathrm{e}, \text { ped }}$ intrinsically decays, ELM suppression is accessed and the edge $V_{\phi}^{C 6+}$ bifurcates to more positive values. The conclusion to be drawn is that while the critical $\omega_{E}$ zero-crossing is a necessary condition for ELM suppression, it is not the only consideration $-n_{\text {e,ped }}$ must also be below threshold. Even with both conditions satisfied there may be a short delay comparable to the energy confinement time scale before robust ELM suppression sets in. This conclusion was also arrived at in previous experiments that rapidly engaged the RMP coils [40]. Note that the high $\Delta_{\text {up }}$ discharges in Section 3 are free of this ambiguity since they all operated well below the higher $n_{\text {e,ped }}$ threshold of that shape.

As a prelude to Section 5, the other consideration in the evolutions of Fig. 11 is the role of the NBI torque. While the evolutions of discharges 174692 and 174693 are very similar, they dynamically access ELM suppression at different $n_{\mathrm{e}, \mathrm{ped}}$, and are differentiated mainly by the applied NBI torque. The higher torque discharge (4.4 vs. 
3.3 Nm) was able to access ELM suppression earlier in time and at slightly higher $n_{\mathrm{e}, \mathrm{ped}}$. These dynamics are rapid and complicated, being also greatly impacted by the ELM cycle dynamics [41, 29]. It is thus more difficult to resolve criticality conditions entering ELM suppression (as in most of the low $\Delta_{\text {up }}$ cases) with as great a precision as the criticality condition for exiting ELM suppression (as in the Sec. 3 cases).

\section{Crossing the Rotation Threshold by Actuating the NBI Torque}

Analysis up to this point has focused on the rotation threshold without consideration of the role of momentum actuators to access and cross this threshold. In this section the observed rotation threshold is considered both in terms of the inherent momentum confinement, the total injected NBI torque, and the edge torque density as computed by the TRANSP code [42], with the latter implicated as a particularly important controlling parameter.

\subsection{Total NBI Torque and Power Operating Space}

As discussed in Section 2, experiments were conducted by varying the NBI injection quantity and geometry. Figure 12 demonstrates the accessed total NBI power and torque in both plasma shapes, with ELM suppressed time-slices indicated in magenta. Using only co- $I_{P}$ directed NBI (hereafter co-NBI), only a narrow NBI operating space is accessible, as defined by the dashed lines. Variations within the co-NBI only operating space are possible by changing the ratio of tangentially to radially directed co-NBI sources. Using counter- $I_{P}$ directed NBI (hereafter ctr-NBI), the power is increased but the torque is decreased, significantly expanding the accessible operating space towards the lower right.

Considering results in the $\Delta_{\text {up }} \sim 0.3$ shape [Fig. 12(a)], ELM suppression is found for nearly all combinations of co- $I_{P} \mathrm{NBI}$, regardless of whether the total torque is around $2 \mathrm{Nm}$ (2 co-radial sources) or $6 \mathrm{Nm}$ (3 co-tangential sources). Crossing the rotation threshold in this shape required ctr-NBI injection. ELMing conditions can be encountered at either low $(\approx 3 \mathrm{Nm})$ or high $(\approx 5 \mathrm{Nm})$ total torque, as long as sufficient ctr-NBI is used. However, some amount of ctr-NBI is found to be compatible with ELM suppression as evidenced by the magenta points that exist below the co-radial dashed line. In contrast, the $\Delta_{\text {up }} \sim 0.1$ shape is found to access suppression in a much narrower range of NBI power and torque [Fig. 12(b)]. Specifically, using only co-radial sources is marginal for ELM suppression (mix of both black and magenta dots on the lower dashed line), and all cases with ctr-NBI (below the dashed line) are ELMing. Cases with mostly co-tangential NBI but no ctr-NBI were robustly ELM-suppressed. Note some overlap between ELMing and ELM suppressed data is expected in NBI operating space, due to inherent variations of the rotation at constant NBI mix. 

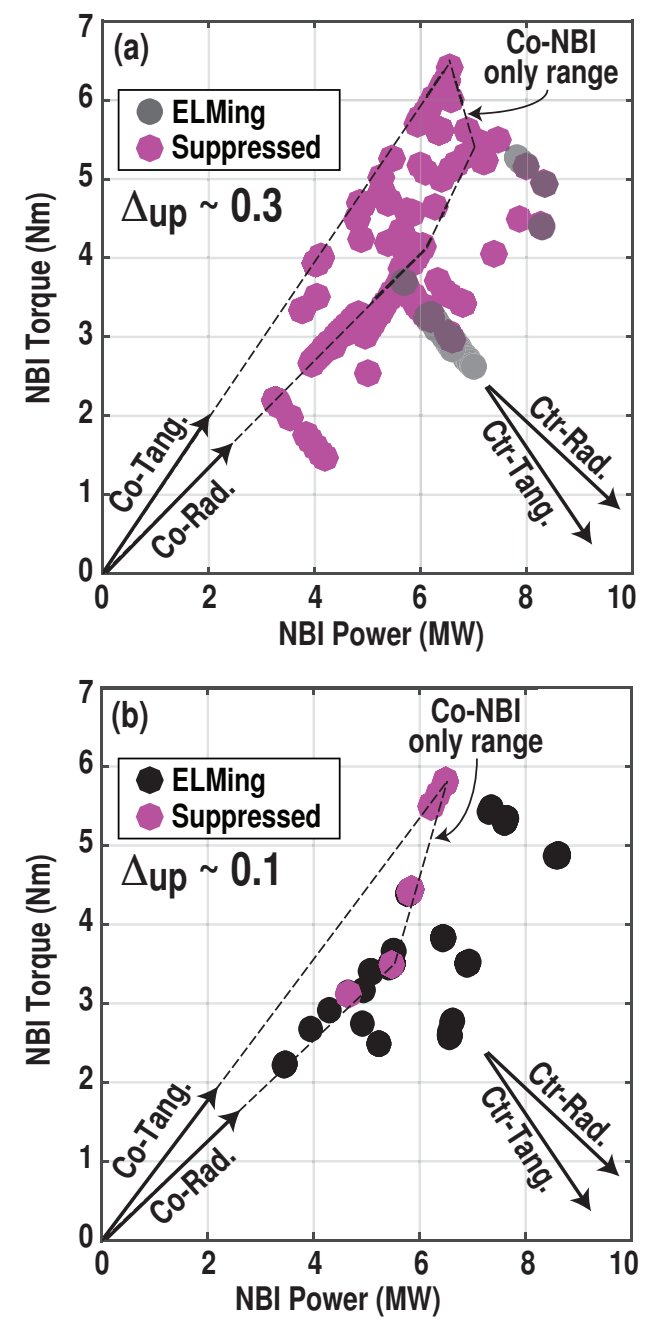

Figure 12. (color online) Operating space for ELM suppression in terms of total NBI power and torque (a) the $\Delta_{\text {up }} \sim 0.3$ shape and (b) the $\Delta_{\text {up }} \sim 0.1$ shape. The range accessed with co-NBI only is indicated by the dashed lines.

\subsection{Differences with Plasma Shape}

The narrower NBI operating space of the low $\Delta_{\text {up }}$ shape can be understood at least in part by considering three features. First, as shown in Sec. 4, the rotation threshold is higher and the critical $\omega_{E}$ radius more outward at $\Delta_{\text {up }} \sim 0.1$.

Second, the reduction of $\Delta_{\text {up }}$ is found to have a significant effect on global energy confinement. Figure 13(a) demonstrates a markedly higher energy confinement time at high $\Delta_{\text {up }}$, as expected from improved pedestal stability at high $\Delta_{\text {up }}$ allowing access to higher $P_{\text {ped }}$ [43]. Higher overall confinement should also be partially responsible for the generally higher edge rotation at the same total injected NBI torque, as shown in Fig. 13 (b).

Third is the expected increase in intrinsic rotation from the higher pedestal ion temperatures $\left(T_{\mathrm{i} \text {,ped }}\right)$ and pressures (shown in Fig. 1) at high $\Delta_{\text {up }}$. The pedestal-top ion temperature (higher at high $\Delta_{\text {up }}$ ) is thought to directly increase the intrinsic rotation 

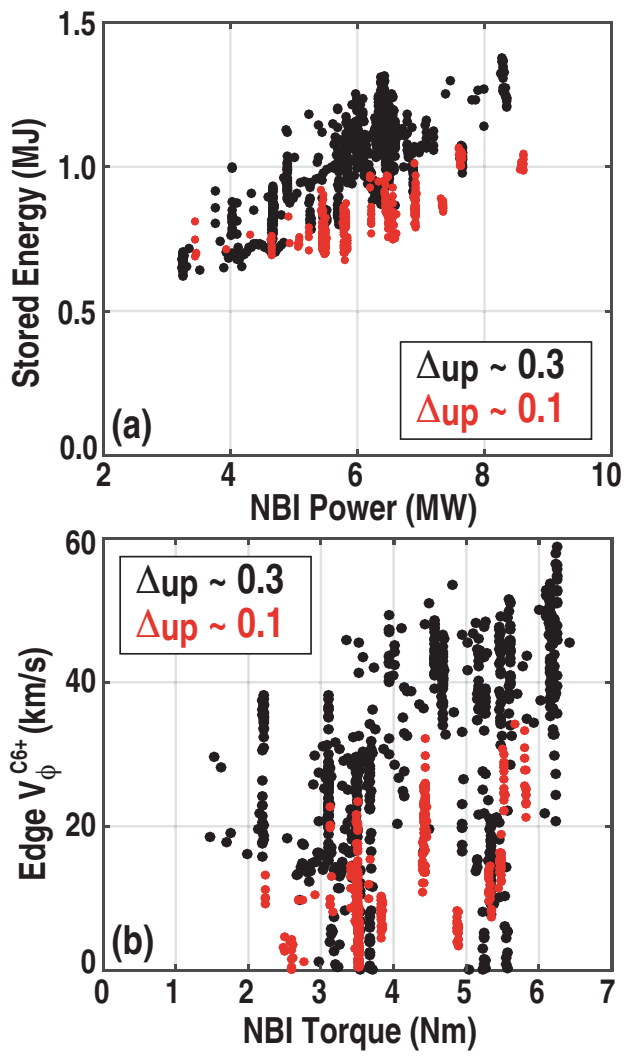

Figure 13. (color online) Comparison of (a) global energy confinement and (b) edge $V_{\phi}^{C 6+}$ vs. NBI torque for both $\Delta_{\text {up }}$.

[44, and studies on DIII-D have found a linear dependency of the intrinsic rotation and torque with $T_{\mathrm{i}, \text { ped }}$ [45] and pedestal pressure gradient [46]. Considering that the characteristic $T_{\mathrm{i} \text {,ped }}$ increases from $1.3 \mathrm{kV}$ to $1.6 \mathrm{kV}$, an increase of $10 \mathrm{~km} / \mathrm{s}$ is expected from $\Delta_{\text {up }} \sim 0.1$ to $\Delta_{\text {up }} \sim 0.3$ using the relationship of Fig. 7 of Ref. [45], explaining some of the increase shown in Fig. 13(b).

These three effects (decreased rotation threshold, increased global confinement, and increased intrinsic rotation) all work together to make the $\Delta_{\text {up }} \sim 0.3$ shape more favorable for ELM suppression. Implications for extrapolations to future tokamaks will be left to Sec. 6.

\subsection{Total NBI Torque vs. Edge NBI Torque Density}

Figure 12 demonstrated a severe penalty to ctr-NBI for ELM suppression, as discharges were more likely to be ELMing if counter-NBI was in the NBI mix despite similar total NBI torque. Results in this section will be considered in terms of the NBI torque density at the plasma edge (averaging from $\rho=0.85$ to 0.95 ), focusing on the $\Delta_{\text {up }} \sim 0.3$ shape. Averaging the NBI torque density over the broad edge region is meant to provide a simple metric that highlights the region of the torque profile that impacts the edge rotation. The transport code TRANSP [42] is used to compute the NBI torque density 


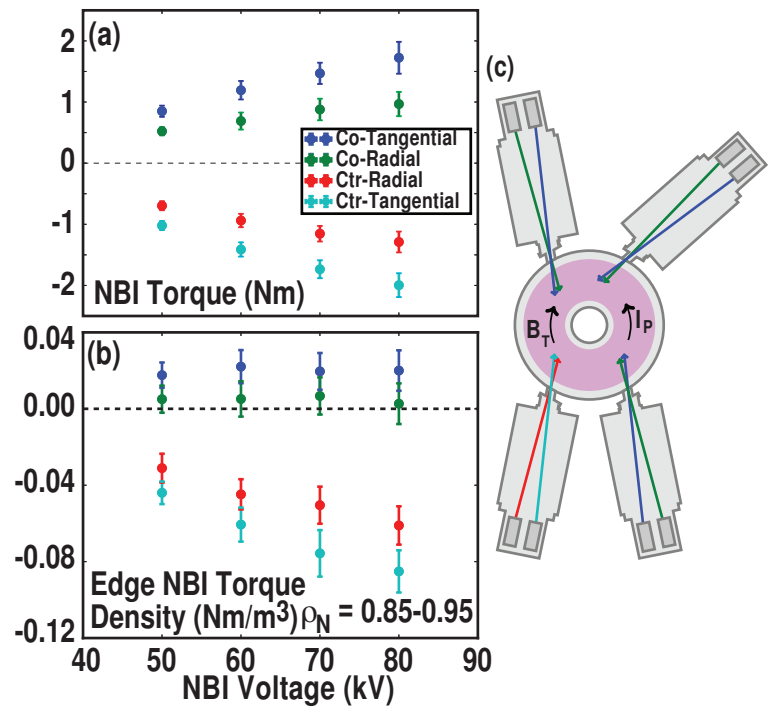

Figure 14. (color online) TRANSP calculation of (a) the total input NBI torque, and (b) the NBI torque density at the edge (averaged from $\rho=0.85$ to 0.95 ) as a function of source voltage and geometry.

profiles for each of the NBI source geometries present on DIII-D, and integrated to give the TRANSP-evaluated total NBI torque per source. These calculations include the collisional and collisionless prompt torque [47]. A representative $\Delta_{\text {up }} \sim 0.3$ equilibrium is used, and the torque profiles were calculated varying the NBI source geometry and voltage, with results shown in Fig. 14. As expected, the total NBI torque is roughly consistent between co- and ctr-NBI sources and increases with NBI voltage. However, the NBI torque density at the edge is severely imbalanced from co- to ctr-NBI, with the ctr-NBI sources roughly $4 \mathrm{x}$ more effective at depositing torque at the edge. The physical mechanism for this discrepancy is the direction the neutral beam ion trapped banana orbit travels after ionization within the plasma. The trajectory is radially outward for counter- $I_{P}$ going particles and radially inwards for co- $I_{P}$ directed particles [47.

These calculations allow a re-mapping of the $\Delta_{\text {up }} \sim 0.3 \mathrm{NBI}$ operating space [Fig. 12 (a)] and rotation threshold [Fig. 2(a)] to the edge NBI torque density, and this is shown in Fig. 15. The relationship between edge NBI torque density and total NBI torque is not co-linear due to the disparate edge NBI torque densities between co- and ctr-NBI.

While no simple torque threshold is found (nor expected), a fairly clear separation between discharges that eventually ELM and those that do not is found by comparing the edge NBI torque density. As shown in Fig. $15(\mathrm{~b})$, all discharges below $\approx-0.02$ $\mathrm{Nm} / \mathrm{m}^{3}$ experience a sufficiently large reduction of edge $V_{\phi}^{C 6+}$ to eventually cross the threshold value and become ELMing. The transition also happened once at higher edge NBI torque density $\left(\approx 0 \mathrm{Nm} / \mathrm{m}^{3}\right)$, indicating that the details of the momentum balance that connects a given torque to the edge $V_{\phi}^{C 6+}$ are varying. It is important to note that this negative NBI torque density level exists despite the fact that total NBI torque is 

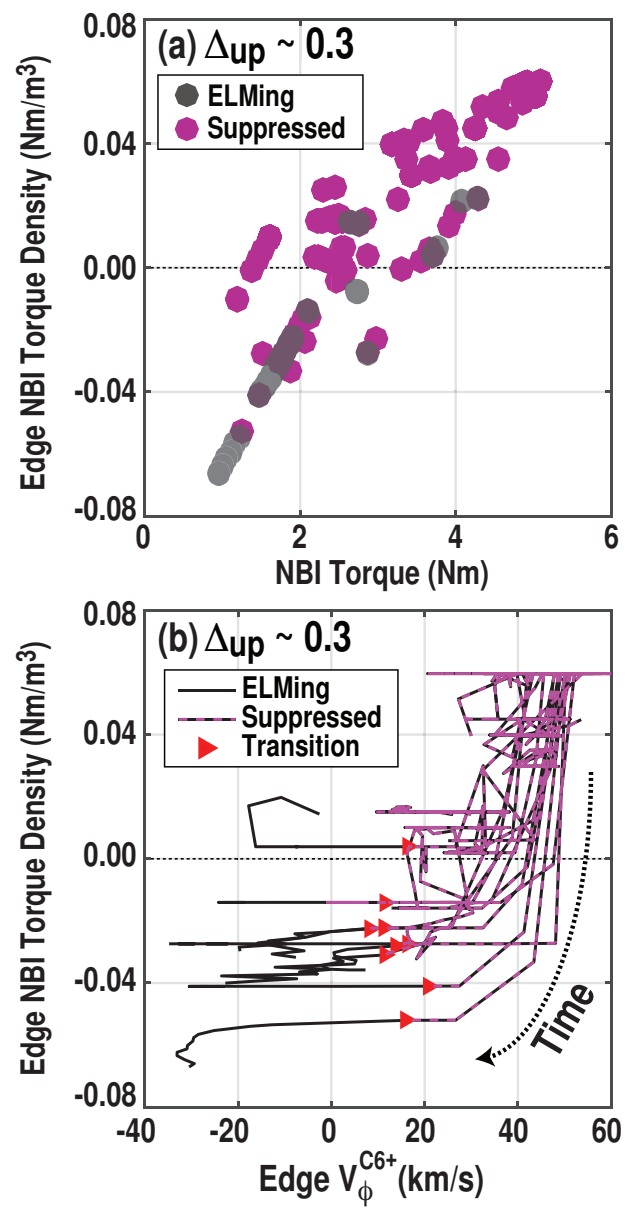

Figure 15. (color online) (a) TRANSP-evaluated NBI torque and (b) edge $V_{\phi}^{C 6+}$ operating space represented in terms of the TRANSP-computed torque density averaged over the edge.

still positive. These observations support a more important role for the edge NBI torque density than the total torque, and demonstrate the severe edge rotation penalty found when using ctr-NBI. Implications for extrapolation will be discussed in Sec. 6 .

\section{Summary and Discussion}

In this work the rotation threshold has been carefully documented in two different plasma shapes with differing $\Delta_{\text {up }}$. Comparisons to both the $\omega_{E}$ and $\omega_{\perp, e}$ zero-crossings find a critical radius for the $\omega_{E}$ location at the exit of ELM suppression. Changing the plasma $\Delta_{\text {up }}$ has a profound impact on the pedestal structure and also changes the operational boundaries for ELM suppression in terms of $n_{\mathrm{e}, \mathrm{ped}}$ and rotation. Several issues raised are now discussed. 


\subsection{Summary of Changes with Triangularity}

Dedicated variation of $\Delta_{\text {up }}$ varied the operating space for ELM suppression significantly. The raw $V_{\phi}^{C 6+}$ rotation threshold was higher at low $\Delta_{\text {up }}$, and corresponded to a more outward critical $\omega_{E}$ zero-crossing location. The reason for this is not known, though a plausible hypothesis is as follows: assuming the region of enhanced transport surrounding the $\omega_{E}$ zero-crossing location is of similar width between shapes, the narrower pedestal of the $\Delta_{\text {up }} \sim 0.1$ shape would require a more outward $\Psi_{N}$ location of the $\omega_{E}$ zero-crossing in order to connect the enhanced transport region to the pedestal top. Given the spacing between rational surfaces (and the slightly different $q_{95}$ at each $\left.\Delta_{\text {up }}\right)$, this equates to the $10 / 3$ surface at $\Delta_{\text {up }} \sim 0.3$ and the $9 / 3$ surface at $\Delta_{\text {up }} \sim 0.1$.

A second observation is that the $n_{\mathrm{e}, \text { ped }}$ threshold falls from $\approx 3$ to $\approx 2 \times 10^{19} \mathrm{~m}^{-3}$ when $\Delta_{\text {up }}$ is reduced from 0.3 to 0.1 . Considering the significantly lower $T_{\text {e,ped }}$ at low $\Delta_{\text {up }}$, this lower threshold is consistent with operating in a similar $\nu_{e}^{*}$ regime in both shapes. That being said, no single threshold in $\nu_{e}^{*}$ is found.

Finally, the required NBI torque for ELM suppression was generally higher at low $\Delta_{\text {up }}$, and observed edge $V_{\phi}^{C 6+}$ was lower at a given NBI torque. This is consistent with both a decrease in the momentum confinement time as well as a smaller intrinsic rotation due to lower $T_{\mathrm{i}, \text { ped }}$.

\subsection{Comparison of Findings with AUG Tokamak}

The variation of $\Delta_{\text {up }}$ was motivated by recent findings of DIII-D/AUG similarity experiments that identified increasing $\Delta_{\text {up }}$ as the essential feature that enabled ELM suppression on AUG [11, 12, 13]. While these experiments were not meant to be rigorous similarity experiments, both devices operate in similar regimes and so some commonality would be expected. A potentially important caveat is that this study uses $n=3$ RMPs while the AUG study used $n=2$. Before discussing ELM suppression, an interesting transport difference is identified. In DIII-D the highest edge $V_{\phi}^{C 6+}$ is seen at low $n_{\text {e,ped}}$, as shown in Fig. 9(b). In contrast, in AUG high $n_{\mathrm{e}, \text { ped }}$ is observed to have the highest impurity rotation (Fig. 8 in Ref. [13]). The origin of this difference is not known.

Considering ELM suppression, a high-level take away is that the reduction of $\Delta_{\text {up }}$ in these experiments reduced the operating space for ELM suppression, broadly consistent with AUG. However, the absence of ELM suppression in AUG at low $\Delta_{\text {up }}$ cannot be simply explained by this argument, since similar $\nu_{e}^{*}$ and $V_{\phi}^{C 6+}$ ranges have been obtained in both low and high $\Delta_{\text {up }}$ shapes. One possibility is that the large $n_{\text {e,ped }}$ threshold reduction with $\Delta_{\text {up }}$ is important separately from $\nu_{e}^{*}$, and as such that the threshold cannot be crossed except at prohibitively low $n_{\mathrm{e}, \mathrm{ped}}$. Considering the absence of a $V_{\phi}^{C 6+}$ threshold reported in Ref. [13], a simple explanation may be that $V_{\phi}^{C 6+}$ cannot be sufficiently reduced without ctr-NBI. However, the reported $\omega_{E}$ zero-crossing location of $\Psi_{N} \approx 0.85$ (inside of the $7 / 2$ surface) is considerably interior from the thresholds identified here $\left(\Psi_{N} \approx 0.92-0.94\right)$. More direct similarity experiments (matching RMP spectrum) may be needed to resolve these discrepancies, though both devices do find 


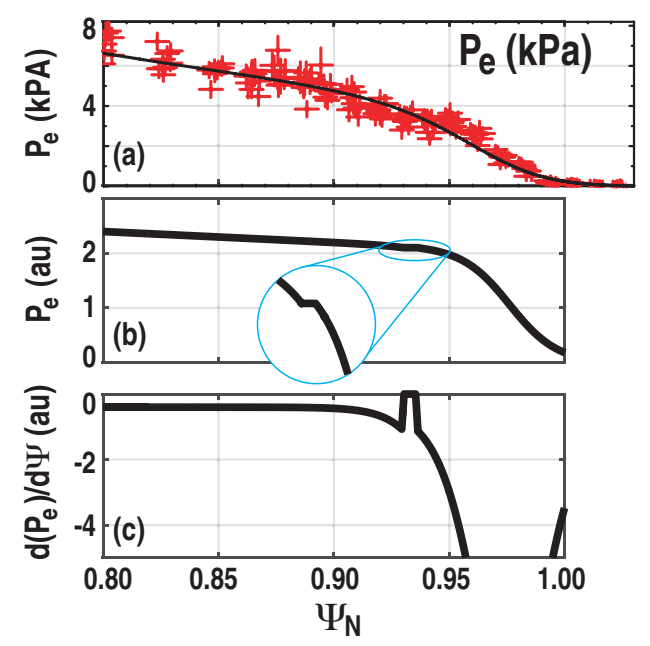

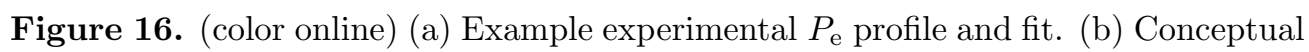
$P_{\mathrm{e}}$ profile with narrow flat-spot. (c) Gradient (proportional to $\omega_{*, C}$ ) for this profile. $\omega_{*, e}=0$ would yield $\omega_{E}=\omega_{\perp, e}$ locally.

that $\omega_{\perp, e}$ can be negative everywhere while maintaining ELM suppression.

\subsection{Role of Small-Scale Flattening in Isolating $\omega_{E}$ and $\omega_{\perp, e}$}

These experimental studies have found a strong correlation of the $\omega_{E}$ zero-crossing with ELM suppression, while no correlation is found for the $\omega_{\perp, e}$ zero-crossing. These results are consistent with an important role for the kinetic resonances for field penetration described in Ref. [30] and challenge two-fluid MHD models for field penetration [28]. However, it is important to stress that the difference between $\omega_{E}$ and $\omega_{\perp, e}$ is the electron diamagnetic flow $\left(\omega_{*, e}\right)$, which is proportional to the electron pressure gradient $\left[d\left(P_{e}\right) / d \Psi\right]$. Figure 16 demonstrates an experimental quandary: if one imagines a narrow region of enhanced transport (due to a penetrated island, for example), a narrow flatspot in the $P_{\mathrm{e}}$ would result [Fig. 16(b)]. This would locally make $\omega_{*, e}=0$, and as such locally $\omega_{E}=\omega_{\perp, e}$. An experimentally measured profile would not be able to resolve such a small feature, as shown in Fig. 16(c), and as such the experiment would interpret $\omega_{E}$ as the essential parameter. This theoretical ambiguity may remain unresolved until measurement accuracy significantly improves at the mm-scale, although $\omega_{E}$ would still be the de-facto controlling parameter. The amount of profile flattening expected for a penetrated island is also not trivial, depending on the inner layer parameters as well as the amount of penetrated field (i.e, the island size) [48. Another interesting question is whether it may be possible to identify a de-facto role for $\omega_{\perp, e}$ in the access to field penetration, prior to the formation of the possible flat-spot, but exclude it at the exit of ELM suppression, when the possible flat-spot would have been present. This possibility may reconcile these findings with previous results identifying the first response of the RMP field at the $\omega_{\perp, e}$ zero-crossing [49]. More experimental data targeted to resolve this difference is needed. 


\subsection{The Prejudicial Role of Counter- $I_{P}$ Directed NBI}

This work has demonstrated a particularly prejudicial role for ctr-NBI in affecting the edge $V_{\phi}^{C 6+}$ and ELM suppression itself. This can be seen by comparing results at the same total NBI torque in Fig. 12. Without ctr-NBI, the edge torque and $V_{\phi}^{C 6+}$ remain high, and ELM suppression remains. With ctr-NBI (at the same total torque), the edge torque can be strongly negative, and the edge $V_{\phi}^{C 6+}$ more susceptible to falling below the threshold. This observation raises important questions about how to accurately simulate ITER discharges in NBI-heated plasmas. While ctr-NBI is an extremely useful and efficient actuator to cross the rotation threshold and exit ELM suppression, the net global NBI torque does not appear to be a relevant metric for extrapolation or simulation of pedestal effects. Said differently, the DIII-D 'ITER-equivalent torque' [50] of $\approx 0.5$ Nm can yield a rather ITER-irrelevant strongly negative edge NBI torque density at the pedestal-top if significant ctr-NBI is in the NBI mix. This study motivates increased emphasis on RF-heating over co + ctr-NBI in DIII-D for pedestal studies.

\subsection{Extrapolation of the Rotation Threshold}

This work has identified several key features of importance to projecting ELM suppression access conditions to future tokamaks such as ITER.

The first is the consideration of the $\omega_{E}$ zero-crossing location. Predictions for the rotation profiles in future tokamaks [51,52] should highlight this profile, and in particular where the zero-crossing is located with respect to the pedestal-top and the edge rational surfaces. Naturally, the impact of the RMP on the rotation profile is also essential to incorporate [53, 54, 55]. This extrapolation is complicated, requiring evaluation of the intrinsic torque as well as the RMP. However, by focusing on edge local torques perhaps some simplifications can result. An additional flexibility not considered here is the role of RMP spectrum. The ITER ELM coils allow excellent spectral flexibility and will afford additional opportunities to localize the RMP-driven torque to the edge of the plasma and encourage field penetration.

A second consideration is an understanding of what determines the critical spacing between the $\omega_{E}$ zero-crossing and the pedestal-top. When spacing between adjacent rational surfaces is not large (as with high $n$ RMPs or high $q_{95}$ operation) it is important to understand why penetration at one rational surface may be sufficient, but a nearby rational surface is not. Answering this question will require further coupling of microscale fluctuation models with global transport models [56, 20] to understand this critical spacing.

It is also worth highlighting that the demonstration of an increased $n_{\text {e,ped }}$ threshold with higher $T_{\text {e,ped }}$ is favorable for ITER, as it suggests a collisionality-based scaling as opposed to a density-based extrapolation. Improved understanding of the density threshold and its relationship to collisionality is needed to better extrapolate this operational boundary. 


\section{Conclusion}

In this paper variation of NBI mix reveals a clear rotation threshold for RMP-ELM suppression that is found to correspond to a critical radius for the $\omega_{E}$ zero-crossing. No such critical location is observed for the $\omega_{\perp, e}$ zero-crossing. NBI mix variations also reveal that the rotation threshold can be crossed at widely varying total injected NBI torques. Computing the local torque density at the edge, the rotation threshold is found to be crossed (from ELM suppression to ELMing) when the local edge NBI torque is negative in all but one discharge (out of nine). Decreasing $\Delta_{\text {up }}$ from 0.3 to 0.1 is found to narrow and lower the pedestal. This in turn: 1) Increases the rotation threshold and yields a more outward critical $\omega_{E}$ zero-crossing location. 2) Decreases the density threshold, consistent with a comparable collisionality range at lower pedestal temperatures. 3) Increases the input torque requirement, due to observed lower confinement and expected smaller intrinsic torque in the lower triangularity shape. These findings represent an important step along the road to predicting ELM suppression access conditions in future tokamaks such as ITER, where the toroidal rotation is expected to be small and consequently the rotation zero-crossings far from the pedestal-top.

\section{Appendix A. Rotation Effect on Pedestal Shape and Potential Alternate Path to ELM Instability}

This appendix will discuss coincidental variations in the pedestal shape and stability as the edge rotation was reduced with counter-NBI while ELM suppression was maintained. While these changes were not found to be essential to the rotation criticality observed, in principle they offer an alternate route to ELMing as the rotation is reduced, and are also of fundamental importance in terms of understanding the pedestal structure. As these effects were only clear with $\Delta_{\text {up }} \sim 0.3$, discussion focuses on that condition.

\section{Appendix A.1. Variation of Pedestal Shape}

Figure A1 presents a detailed evolution of the pedestal profiles as the rotation is reduced with counter-NBI while maintaining ELM suppression in discharge 169476, with the profile colors matching Figs. 4 and 5 to locate the time during the rotation evolution. Several key features are identified as the rotation is reduced.

First, considering the ion channel, the impurity density profile significantly shifts inwards and the gradients increase as the rotation is reduced, resulting in a larger carbon diamagnetic flow in the negative direction. Noting the constant $\omega_{E}$ zero-crossing in Fig.

4. the $\omega_{*, C}$ changes happen in parallel with $V_{\phi}^{C 6+}$ changes that together cancel out any effect on the $\omega_{E}$ zero-crossing. Only very minor changes are observed in the ion temperature $\left(T_{\mathrm{i}}\right)$.

Considering the electron channel, as the rotation is reduced a significant increase in the $T_{\text {e,ped }}$ alongside a narrowing of the electron pedestal width, as shown in Fig. 

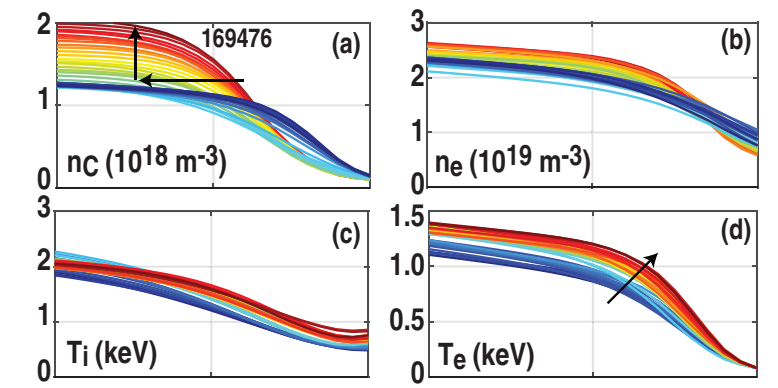

(c)
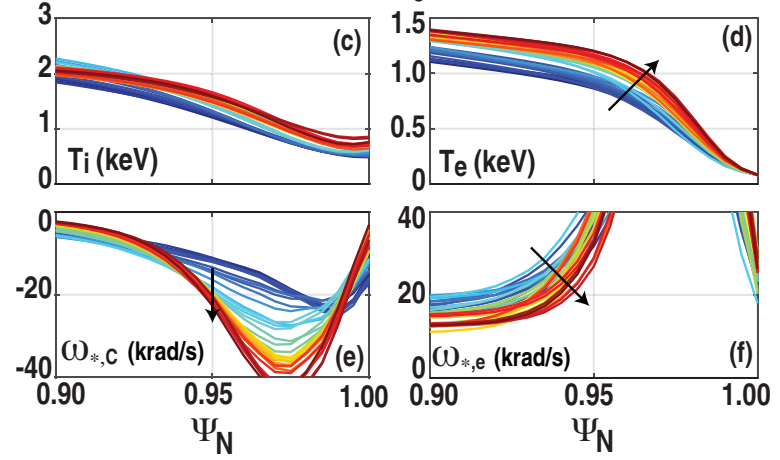

Figure A1. (color online) Variation of the pedestal profiles as rotation is reduced with counter-NBI while ELM suppression is maintained, in terms of (a) carbon impurity density, (b) electron density, (c) ion temperature, (d) electron temperature, (e) carbon diamagnetic rotation, (f) electron diamagnetic rotation.

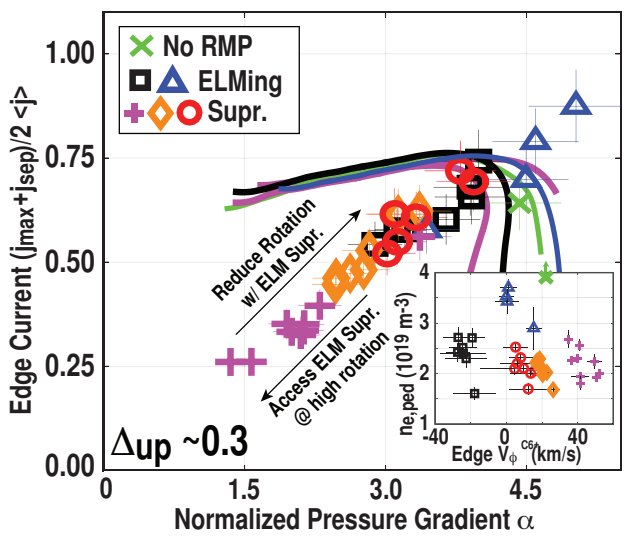

Figure A2. (color online) Pedestal stability calculations using the ELITE code for the $\Delta_{\text {up }} \sim 0.3$ shape. The colors match profile parameters in the inset.

A1(d). This effect is also clear from examining the operating space plot of Fig. 9(c). The reason this occurs is not understood. The poloidal beta (and thus Shafranov shift) in this discharge is decreasing, ruling out pedestal stabilization via this effect. The discharges that exhibit this effect necessarily have counter-NBI to sufficiently reduce the edge rotation, opening the possibility for a direct effect of this actuator perhaps through modifications of the electric field through orbit effects. While these changes occur too radially-outward to affect the $\omega_{\perp, e}$ zero-crossing, they do severely affect the pedestal stability, as will now be discussed. 


\section{Appendix A.2. Impact on Pedestal Stability}

A comparison of the pedestal stability across the ELM suppression operating space of Fig. 10 was conducted using the ELITE code that calculates the instability threshold of the peeling-ballooning modes that are thought to non-linearly give rise to the ELM [57, 58]. The pedestal shape effects discussed in Appendix A.1 result in significant pedestal stability changes as the rotation is varied, as shown in Fig. A2. The color coding is provided to locate profiles in the functional operating space of $n_{\mathrm{e} \text {,ped }}$ and edge $V_{\phi}^{C 6+}$ using the inset.

Discharges operate over a wide swath of pedestal stability space. Broadly speaking, ELMing discharges operate closer to the stability boundary than ELM suppressed discharges. The stability boundary does not exhibit large changes for the cases studied. Within ELMing profiles, low density and low rotation cases (black) tend to have weaker gradients then the high-density cases (blue).

A key observation is that the pedestal stability within the ELM suppressed dataset varies considerably. The rotation is found to be a strong determinate of the operating point of the ELM suppressed pedestal. As such, several additional colors (red, orange, magenta) are used to differentiate between ELM suppressed profiles at different rotation levels. The highest rotation cases (magenta) are most firmly in the stable part of the pedestal stability space. As rotation is reduced, the steepening of the $T_{\text {e }}$ profile shown in Fig. A1 (d) results in a higher peak pressure gradient $(\alpha)$ and associated bootstrap current. At the limit of the lowest rotations accessed in ELM suppression, the pedestal stability is just below that of the ELMing cases.

This naturally leads to the question of whether the transition to ELMing can be understood simply in terms of the pedestal stability. In these experiments, this is unlikely to be the case for two reasons. First, no critical $T_{\text {e,ped }}$ or $P_{\text {e,ped }}$ is found at the rotation threshold [Fig. 9(c)]. Second, carefully examining the time-history of the $D_{\alpha}$ recycling light reveals a drop in the $D_{\alpha}$ baseline level alongside the rotation preceding the ELM, indicating the enhanced transport from the ELM suppression is lost [16] before the ELM returns. Third, this effect was not readily identifiable in the $\Delta_{\text {up }} \sim 0.1$ dataset, yet it too has a rotation threshold.

To conclude this Appendix, significant variations of the pedestal shape in the $\Delta_{\text {up }} \sim 0.3$ condition were found as rotation was reduced while maintaining ELM suppression. This significantly affected the pedestal stability operating point, with the lowest rotation points operating close to the ELMing points. This mechanism (of uncertain origin) may provide a potentially important alternate path to ELM instability as the rotation is reduced. Even with the additional transport from fully penetrated RMPs, the pedestal may still cross the peeling-ballooning stability limit at low rotation. This would require yet another mechanism to further increase the transport and avoid the ELM. As extrapolation for this effect is unclear, this mechanism may define the operating space more than the $\omega_{E}$ zero-crossing location in some conditions. In these discharges, however, ELM suppression (evidenced by enhanced $D_{\alpha}$ light and exterior 
$\omega_{E}$ zero-crossing) is lost before the ELM onsets, so this alternate path to instability was not the limiting factor.

\section{Acknowledgments}

The authors would like to thank A. Hyatt and T. Wilks for operations support, B. Grierson for TRANSP support, S. Haskey, L. Schmitz and G. McKee for diagnostic support, as well as M. Beidler, C. Callen, C. Chrystal, T. Evans, and R. Moyer for useful discussions. This material is based upon work supported by the U.S. Department of Energy, Office of Science, Office of Fusion Energy Sciences, using the DIII-D National Fusion Facility, a DOE Office of Science user facility, under Awards DE-FC02-04ER54698. DIII-D data shown in this paper can be obtained in digital format by following the links at https://fusion.gat.com/global/D3D_DMP. Disclaimer: This report was prepared as an account of work sponsored by an agency of the United States Government. Neither the United States Government nor any agency thereof, nor any of their employees, makes any warranty, express or implied, or assumes any legal liability or responsibility for the accuracy, completeness, or usefulness of any information, apparatus, product, or process disclosed, or represents that its use would not infringe privately owned rights. Reference herein to any specific commercial product, process, or service by trade name, trademark, manufacturer, or otherwise does not necessarily constitute or imply its endorsement, recommendation, or favoring by the United States Government or any agency thereof. The views and opinions of authors expressed herein do not necessarily state or reflect those of the United States Government or any agency thereof.

\section{References}

[1] Loarte A, et al Progress in the ITER Physics Basis Chapter 4: Power and particle control 2007 Nuclear Fusion 47 S203 URL https://doi.org/10.1088/0029-5515/47/6/S04

[2] Loarte A, Huijsmans G T A, Futatani S, Baylor L R, Evans T E, Orlov D M, Schmitz O, Becoulet M, Cahyna P, Gribov Y, Kavin A, Naik A S, Campbell D J, Casper T A, Daly E, Frerichs H, Kischner A, Laengner R, Lisgo S, Pitts R A, Saibene G and Wingen A 2014 Nuclear Fusion 54 033007 ISSN 0029-5515 URL https://doi.org/10.1088/0029-5515/54/3/033007

[3] Evans T E, Moyer R A, Thomas P R, Osborne T H, Boedo J A, Doyle E J, Fenstermacher M E, Finken K H, Groebner R J, Groth M, Harris J H, La Haye R J, Lasnier C J, Masuzaki S, Ohyabu N, Pretty D G, Rhodes T L, Reimerdes H, Rudakov D L, Schaffer M J, Wang G and Zeng L 2004 Physical Review Letters 92235003 URL http://link.aps.org/doi/10.1103/ PhysRevLett.92.235003

[4] Suttrop W, Eich T, Fuchs J C, Günter S, Janzer a, Herrmann a, Kallenbach a, Lang P T, Lunt T, Maraschek M, McDermott R M, Mlynek a, Pütterich T, Rott M, Vierle T, Wolfrum E, Yu Q, Zammuto I and Zohm H 2011 Physical Review Letters 106225004 ISSN 0031-9007 URL http://link.aps.org/doi/10.1103/PhysRevLett.106.225004

[5] Jeon Y M, Park J K, Yoon S W, Ko W H, Lee S G, Lee K D, Yun G S, Nam Y U, Kim W C, Kwak J G, Lee K S, Kim H K and Yang H L 2012 Physical Review Letters 109035004 ISSN 0031-9007 URL http://link.aps .org/doi/10.1103/PhysRevLett.109.035004 
Shape and NBI Effects on the RMP-ELM Suppression Rotation Threshold

[6] Kirk A, Chapman I T, Liu Y, Cahyna P, Denner P, Fishpool G M, Ham C J, Harrison J R, Liang Y, Nardon E, Saarelma S, Scannell R and Thornton A 2013 Nuclear Fusion 53043007 ISSN 0029-5515 URL https://doi.org/10.1088/0029-5515/53/4/043007

[7] Kirk A, Suttrop W, Chapman I T, Liu Y, Scannell R, Thornton A, Orte L B, Cahyna P, Eich T, Fischer R, Fuchs C, Ham C J, Harrison J R, Jakubowski M W, Kurzan B, Pamela S, Peterka M, Ryan D A, Saarelma S, Sieglin B, Valovic M and Willensdorfer M 2015 Nuclear Fusion 55 043011 ISSN 0029-5515 URL https://doi.org/10.1088/0029-5515/55/4/043011

[8] Wade M R, Nazikian R, DeGrassie J S, Evans T E, Ferraro N M, Moyer R A, Orlov D M, Buttery R J, Fenstermacher M E, Garofalo A M, Lanctot M J, McKee G, Osborne T H, Shafer M, Solomon W M, Snyder P B, Suttrop W, Wingen A, Unterberg E A and Zeng L 2015 Nuclear Fusion 55023002 URL http://stacks.iop.org/0029-5515/55/i=2/a=023002

[9] Sun Y, Liang Y, Liu Y, Gu S, Yang X, Guo W, Shi T, Jia M, Wang L, Lyu B, Zhou C, Liu A, Zang Q, Liu H, Chu N, Wang H H, Zhang T, Qian J, Xu L, He K, Chen D, Shen B, Gong X, Ji X, Wang S, Qi M, Song Y, Yuan Q, Sheng Z, Gao G, Fu P and Wan B 2016 Physical Review Letters 117 115001 ISSN 0031-9007 URL http://link.aps.org/doi/10.1103/PhysRevLett.117.115001

[10] In Y, Park J K, Jeon Y M, Kim J, Park G Y, Ahn J W, Loarte A, Ko W H, Lee H H, Yoo J W, Juhn J W, Yoon S W and Park H K 2017 Nucl. Fusion 57116054 URL https: //doi.org/10.1088/1741-4326/aa791c

[11] Nazikian R, et al. "First Observation of ELM Suppression in ASDEX-Upgrade In A Similarity Experiment With DIII-D". Preprint: 2016 IAEA Fusion Energy Conference, Kyoto, PD/1-1 URL https://nucleus.iaea.org/sites/fusionportal/Shared\%20Documents/FEC\%202016/ fec2016-preprints/preprint0952.pdf

[12] Suttrop W, Kirk A, Nazikian R, Leuthold N, Strumberger E, Willensdorfer M, Cavedon M, Dunne M, Fischer R, Fietz S, Fuchs J C, Liu Y, McDermott R M, Orain F, Ryan D A and Viezzer E 2017 Plasma Physics and Controlled Fusion 59014049 ISSN 0741-3335 URL https://doi.org/10.1088/0741-3335/59/1/014049

[13] Suttrop W, Kirk A, Bobkov V, Cavedon M, Dunne M, McDermott R M, Meyer H, Nazikian R, Paz-Soldan C, Ryan D A, Viezzer E, Willensdorfer M, Team t A U and Team t t M 2018 Nuclear Fusion 58096031 URL https://doi.org/10.1088/1741-4326/aace93

[14] Fitzpatrick R 1993 Nuclear Fusion 33 1049-1084 ISSN 0029-5515 URL http://www. iop.org/EJ/ abstract/0029-5515/33/7/I08/

[15] Nazikian R, Paz-Soldan C, Callen J D, DeGrassie J S, Eldon D, Evans T E, Ferraro N M, Grierson B A, Groebner R J, Haskey S R, Hegna C C, King J D, Logan N C, Mckee G R, Moyer R A, Okabayashi M, Orlov D M, Osborne T H, Park J K, Rhodes T L, Shafer M W, Snyder P B, Solomon W M, Strait E J and Wade M R 2015 Physical Review Letters 114105002 URL https://doi.org/10.1103/PhysRevLett.114.105002

[16] Paz-Soldan C, et al "Optimization of the Plasma Response for the Control of EdgeLocalized Modes with 3D Fields". Preprint: 2016 IAEA Fusion Energy Conference, Kyoto, EX2-28 URL https://nucleus.iaea.org/sites/fusionportal/SharedDocuments/ FEC2016/fec2016-preprints/preprint0560.pdf

[17] Lyons B C, Ferraro N M, Paz-Soldan C, Nazikian R and Wingen A 2017 Plasma Physics and Controlled Fusion 59044001 ISSN 0741-3335 URL https://doi.org/10.1088/1361-6587/ aa5860

[18] Gu S, Sun Y, Paz-Soldan C, Nazikian R, Jia M, Wang H H, Guo W , Liu Y Q, Abrams T, Cui L, Evans T, Garofalo A, Gong X, Logan N C, Munaretto S, Orlov D and Shi T 2019 Nuclear Fusion 59026012 URL https://doi.org/10.1088/1741-4326/aaf5a3

[19] Sung C, Wang G, Rhodes T L, Smith S P, Osborne T H, Ono M, Mckee G R, Yan Z, Groebner R J, Davis E M, Zeng L, Peebles W A and Evans T E 2017 Physics of Plasmas 24112305 ISSN 10897674 URL https://doi.org/10.1063/1.4999785

[20] Taimourzadeh S, Shi L, Lin Z, Nazikian R, Holod I and Spong D A 2019 Nuclear Fusion 59046005 URL https ://doi .org/10.1088/1741-4326/aafe3a 
[21] Paz-Soldan C, Nazikian R, Haskey S R, Logan N C, Strait E J, Ferraro N M, Hanson J M, King J D, Lanctot M J, Moyer R A, Okabayashi M, Park J K, Shafer M W and Tobias B J 2015 Physical Review Letters 114105001 URL https://doi.org/10.1103/PhysRevLett.114.105001

[22] Ryan D A, Liu Y Q, Kirk A, Suttrop W, Dudson B, Dunne M and Willensdorfer M 2018 Plasma Physics and Controlled Fusion 60 ISSN 13616587 URL https://doi.org/10.1088/1361-6587/ aab873

[23] Kirk A, et al, 2017 Nuclear Fusion 57102007 URL https://doi.org/10.1088/1741-4326/ aa65e0

[24] Park J K, Jeon Y M, In Y, Ahn J W, Nazikian R, Park G Y, Kim J H, Lee H H, Ko W H, Kim H S, Logan N C, Wang Z, Feibush E A, Menard J E and Zarnstorff M C 2018 Nature Physics URL https://doi.org/10.1038/s41567-018-0268-8

[25] Liu Y, Kirk A, Li L, In Y, Nazikian R, Sun Y, Suttrop W, Lyons B, Ryan D A, Wang S, Yang X and Zhou L 2017 Physics of Plasmas 24056111 ISSN 1070-664X URL http: //aip.scitation.org/doi/10.1063/1.4978884

[26] Moyer R A, Paz-Soldan C, Nazikian R, Orlov D M, Ferraro N M, Grierson B A, Knölker M, Lyons B C, McKee G R, Osborne T H, Rhodes T L, Meneghini O, Smith S P, Evans T E, Fenstermacher M E, Groebner R J, Hanson J M, La Haye R J, Luce T C, Mordijck S, Solomon W M, Turco F, Yan Z and Zeng L 2017 Physics of Plasmas 24102501 ISSN 1070-664X URL http://aip.scitation.org/doi/10.1063/1.5000276

[27] Ferraro N M 2012 Physics of Plasmas 19 056105 ISSN 1070664X URL http://link.aip.org/ link/PHPAEN/v19/i5/p056105/s1\{\&\}Agg=doi

[28] Waelbroeck F L, Joseph I, Nardon E, Bécoulet M and Fitzpatrick R 2012 Nuclear Fusion 52 074004 ISSN 0029-5515

[29] Callen J D, et al, 2016 UW CPTC Report 16-4 URL https://cptc.wiscweb.wisc.edu/ wp-content/uploads/sites/327/2017/09/UW-CPTC_16-4.pdf

[30] Heyn M F, Ivanov I B, Kasilov S V, Kernbichler W, Leitner P, Nemov V V and Suttrop W 2014 Nuclear Fusion 54064005 ISSN 17414326 URL https://doi.org/10.1088/0029-5515/54/6/ 064005

[31] Orlov D M, Moyer R A, Evans T E, Paz-Soldan C, Ferraro N M, Nazikian R, DeGrassie J S, Grierson B A, Eldon D, Fenstermacher M, King J D, Logan N C, Lanctot M J, Maingi R, Snyder P B, Strait E J and Wingen A 2016 Nuclear Fusion 56036020 ISSN 0029-5515 URL https://doi.org/10.1088/0029-5515/56/3/036020

[32] Wilcox R S, Shafer M W, Ferraro N M, McKee G, Zeng L, Rhodes T, Canik J M, Paz-Soldan C, Nazikian R and Unterberg E A 2016 Physical Review Letters 117135001 ISSN 0031-9007 URL http://link.aps.org/doi/10.1103/PhysRevLett.117.135001

[33] Pace D C, Collins C S, Crowley B, Grierson B A, Heidbrink W W, Pawley C, Rauch J, Scoville J T, Van Zeeland M and Zhu Y B 2017 Nuclear Fusion 57014001 ISSN 17414326 URL https://doi.org/10.1088/0029-5515/57/1/014001

[34] Pace D C, Austin M E, Bardoczi L, Collins C, Crowley B, Davis E, Du X, Ferron J, Grierson B A, Heidbrink W, Holcomb C T, McKee G, Pawley C, Petty C, Podestà M, Rauch J, Scoville J, Spong D A, Thome K E, Van Zeeland M, Varela J and Victor B 2018 Physics of Plasmas 25 056109 ISSN 10897674 URL https://doi.org/10.1063/1.5016160

[35] Logan N C, Grierson B A, Haskey S R, Smith S P, Meneghini O and Eldon D 2018 Fusion Science and Technology $\mathbf{7 4} 125$ ISSN 1536-1055 URL https://www.tandfonline.com/doi/full/10. $1080 / 15361055.2017 .1386943$

[36] Leonard A W, McLean A G, Makowski M A and Stangeby P C 2017 Nuclear Fusion 57086033 ISSN 17414326 URL https://doi.org/10.1088/1741-4326/aa778c

[37] Osborne T H, Jackson G L, Yan Z, Maingi R, Mansfield D K, Grierson B A, Chrobak C P, McLean A G, Allen S L, Battaglia D J, Briesemeister A R, Fenstermacher M E, McKee G R and Snyder P B 2015 Nuclear Fusion 55063018 ISSN 0029-5515 URL https://doi.org/10. 1088/0029-5515/55/6/063018 
[38] Lyons B C, Paz-Soldan C, Meneghini O, Lao L L, Weisberg D B, Belli E A, Evans T E, Ferraro N M and Snyder P B 2018 Physics of Plasmas 25056111 ISSN 10897674 URL https://doi.org/10.1063/1.5025838

[39] Sauter O, Angioni C and Lin-Liu Y R 1999 Physics of Plasmas 62834 URL https://doi.org/ $10.1063 / 1.873240$

[40] Orlov D M, Moyer R A, Evans T E, Mordijck S, Osborne T H, Fenstermacher M E, Snyder P B and Unterberg E A 2010 Nuclear Fusion 50034010 ISSN 00295515 URL https://doi.org/ 10.1088/0029-5515/50/3/034010

[41] Beidler M T, Callen J D, Hegna C C and Sovinec C R 2017 Physics of Plasmas 24052508 ISSN 10897674 URL http://dx.doi.org/10.1063/1.4982814

[42] Hawryluk R J 1981 Physics of plasmas close to thermonuclear conditions (Brussels: CEC/Pergamon) ISBN 978-1-4832-8385-2 URL https://doi.org/10.1016/C2013-1-15216-1

[43] Doyle E J, E J Doyle et al. Progress in the ITER Physics Basis Chapter 2: Plasma confinement and transport 2007 Nuclear Fusion 47 S18 URL https://doi.org/10.1088/0029-5515/47/6/S02

[44] Stoltzfus-Dueck T 2012 Physical Review Letters 108 1-5 ISSN 00319007 URL https://doi.org/ 10.1103/PhysRevLett.108.065002

[45] Degrassie J S, Groebner R J, Burrell K H and Solomon W M 2009 Nuclear Fusion 49 ISSN 00295515 URL https://doi.org/10.1088/0029-5515/49/8/085020

[46] Solomon W M, Burrell K H, DeGrassie J S, Boedo J A, Garofalo A M, Moyer R A, Muller S, Petty C C and Reimerdes H 2011 Nuclear Fusion 51073010 ISSN 0029-5515 URL https: //doi.org/10.1088/0029-5515/51/7/073010

[47] DeGrassie J S, Groebner R J and Burrell K H 2006 Physics of Plasmas 13112507 ISSN 1070664X URL http://link.aip.org/link/PHPAEN/v13/i11/p112507/s1\{\&\}Agg=doi

[48] Waelbroeck F L 2009 Nuclear Fusion 49104025 ISSN 0029-5515 URL https://doi.org/10. 1088/0029-5515/49/10/104025

[49] Xiao W W, Evans T E, Tynan G R and Eldon D 2016 Nuclear Fusion 56064001 ISSN 17414326 URL https://doi.org/10.1088/0029-5515/56/6/064001

[50] Garofalo A M, Solomon W M, Park J K, Burrell K H, DeBoo J C, Lanctot M J, McKee G R, Reimerdes H, Schmitz L, Schaffer M J and Snyder P B 2011 Nuclear Fusion 51083018 URL https://doi.org/10.1088/0029-5515/51/8/083018

[51] Chrystal C, Grierson B A, Staebler G M, Petty C C, Solomon W M, DeGrassie J S, Burrell K H, Tala T and Salmi A 2017 Physics of Plasmas 24056113 ISSN 10897674 URL https: //doi.org/10.1063/1.4979194

[52] Grierson B A, Staebler G M, Solomon W M, McKee G R, Holland C, Austin M E, Marinoni A, Schmitz L, Pinsker R I and Team D D 2018 Physics of Plasmas 25022509 ISSN 10897674 URL https://doi.org/10.1063/1.5011387

[53] Liu Y, Akers R, Chapman I T, Gribov Y, Hao G Z, Huijsmans G T A, Kirk A, Loarte A, Pinches S D, Reinke M, Ryan D A, Sun Y and Wang Z R 2015 Nuclear Fusion 55063027 ISSN 0029-5515 URL https://doi.org/10.1088/0029-5515/55/6/063027

[54] Park J K and Logan N C 2017 Physics of Plasmas 032505032505 URL http://dx.doi.org/10. 1063/1.4977898

[55] Logan N C, Park J K, Paz-Soldan C, Lanctot M J, Smith S P and Burrell K H 2016 Nuclear Fusion 56036008 ISSN 0029-5515 URL http://dx.doi.org/10.1088/0029-5515/56/3/036008

[56] Holod I, Lin Z, Taimourzadeh S, Nazikian R, Spong D A and Wingen A 2017 Nuclear Fusion 57 016005 ISSN 0029-5515 URL http://dx.doi.org/10.1088/0029-5515/57/1/016005

[57] Wilson H R, Snyder P B, Huysmans G T A and Miller R L 2002 Physics of Plasmas 91277 ISSN 1070664X URL https://doi.org/10.1063/1.1459058

[58] Snyder P B, Wilson H R, Ferron J R, Lao L L, Leonard A W, Osborne T H, Turnbull a D, Mossessian D, Murakami M and Xu X Q 2002 Physics of Plasmas 92037 ISSN 1070664X URL http://dx.doi.org/10.1063/1.1449463 